\title{
Eigenstates and Absorption Spectra of Interdiffused AlGaAs-GaAs Multiple-Quantum-Well Structures
}

\author{
E. Herbert Li, Senior Member, Bernard L. Weiss, Senior Member, IEEE, \\ and Kwok-Sum Chan, Member, IEEE
}

\begin{abstract}
We present a comprehensive analysis of the interwell coupled eigenstates in interdiffused AIGaAs-GaAs multiplequantum-well structures. A full numerical calculation is considered without any approximation or presumption of the eigenstates. During the initial interdiffusion in the quantum-well structure with well and barrier thickness equaling $100 \AA$, the wavefunctions behave as in the case of a single quantum well with almost no well-to-well coupling of the states. However, as interdiffusion proceeds, the eigenstate in each subband forms minibands, as in the case of superlattice. Distortion of the coupled wavefunctions can also be observed as a consequence of the nonuniformity of the confined wells at the far sides of the multiquantum-well core. The polarized absorption coefficients, including valence band-mixing, are also calculated. Results show that the blue shift of the absorption edge is greater in the range of $10 \AA \leq L_{d} \leq 30 \AA$. The two-dimensional quantum-well properties are at its strongest in the beginning of interdiffusion. An estimation of the modulator performance also shows an improvement of the contrast ratio and lower absorption loss during the initial stage of interdiffusion. This predicts wavelength tuning range of almost $60 \mathrm{~nm}$.
\end{abstract}

\section{INTRODUCTION}

$\mathbf{I}^{\mathbf{r}}$ NTERDIFFUSED nonsquare single- and multiplequantum-well structures are currently attracting considerable interest for their applications in optoelectronics [1]. This stems from both a fundamental point of view as well as from the realization of high-performance devices and the fabrication of optoelectronic integrated circuits [2]. The interdiffusion process ${ }^{1}$ is produced by using post-growth thermal annealing to induce the solid-state interdiffusion of atoms (such as $\mathrm{Al}$ and $\mathrm{Ga}$ ) through the quantum-well (QW) heterointerfaces (such as AlGaAs-GaAs) which alters the

Manuscript received November 22, 1995; revised April 22, 1996. This work was supported in part by the HKU-CRCG grant, the CityUHK strategic research grant, the European Research Office of the U.S. Army, and Defense Advanced Research Projects Agency.

E. H. Li is with the Department of Electrical and Electronic Engineering, University of Hong Kong, Hong Kong.

B. L. Weiss is with the Department of Electronic and Electrical Engineering, University of Surrey, Guildford, Surrey, GU2 5XH, U.K.

K.-S. Chan is with the Department of Physics and Materials Science, City University of Hong Kong, Kowloon, Hong Kong.

Publisher Item Identifier S 0018-9197(96)05595-9.

${ }^{1}$ It should be noted that in the literature the QW material modified by thermal interdiffusion with or without the introduction of intentional impurities is synonymously termed as "mixing" and "disordering." alloy-composition profile (such as the spatial profile of the Al-mole fraction along the QW growth axis) across the QW structure. In addition, the interdiffusion rate can be controlled by the techniques of impurity-induced disordering [3] and impurity-free vacancy interdiffusion. ${ }^{2}$ The optical properties of the quantum well, such as absorption and refractive index, can be modified by the process of interdiffusion (due to band structure changes) so that it provides the ability to tune the operating wavelengths, reduce the power consumption, and increase the switching time of photonic devices. It is also possible to confine photons between layers of as-grown and interdiffused QW's which have different refractive indexes, which is due to the fact [4] that both the band-gap and subband structure of the III-V semiconductor QW are a nonlinear function of the averaged alloy composition. This latter ability to guide light both laterally and vertically in a planar structure is crucial to the monolithic integration technology of optoelectronic integrated circuits and has been demonstrated individually, using selective interdiffusion, in discrete devices, such as lasers [5], waveguides [6], and modulators [7] with some success. However, the modified electronic and optical properties due to this nonsquare well shape are not yet fully understood.

Recent theoretical studies on the interdiffused QW structures consist of the following.

1) Single $Q W$ with infinitely thick barriers: two confinement profiles have been used, one being the error function that was proposed originally to study the interdiffused AlGaAs-GaAs QW band-gap in photoluminescence experiments [8], [9]. The error-function profile is determined from a one-dimension interdiffusion model with a constant interdiffusion coefficient and is used to solve for the lowest subband-edge energy by a variation method with simple trial wavefunctions and without the excitonic effects. Since then, extensive modeling of the subband structures and density of states has been calculated using a finite difference method [10] which has been applied to study the optical properties of interdiffused QW's, including excitons [11]-[15]. The other profile studied is a modified hyperbolic-function approximation where the confined eigenstates are solved

\footnotetext{
${ }^{2}$ This is sometimes known as "bowing" in the literature.
} 
analytically [16], [17]. Although this model is limited to exclude the very early stages of interdiffusion, it has the advantage that it can handle many subbands such as introduced by interdiffusion and has been applied to determine the optical properties of interdiffused QW's [18].

2) Single $Q W$ with finite-thickness barriers: this is a resonance-tunneling type of structure and is therefore ideal for solving the transmission tunneling method. This structure has been used for the calculation of interband and intersubband transitions in interdiffused QW's [19]-[22]. The tunneling method has the advantage of being convenient for the determination of the electric-field-induced absorption broadening and carrier tunneling time.

3) Multiple QW (MQW) and superlattice structures: in addition to an earlier study of the ground-state energy of a superlattice structure [23], there is a more recent report using a five-period MQW of equal as-grown well and barrier widths of $60 \AA$ to study the refractive index around the band-edge [24]. In the latter paper, the experimental fit was reasonably good and nonparabolic band structure was considered for the calculation of subbands, although the properties of subband due to the MQW structure, such as interwell coupling were not discussed. The effects of cladding layer were not considered by either of the above studies. To date, there have been no reports of the subband properties of interdiffused MQW structures which is crucial to the understanding of the operation of optoelectronic devices and how to optimize their performance. Interdiffusion in strain layer QW's is also of interests but is not considered here.

In terms of the effect and process of interdiffusion on device impact, in particular to how modulator performance changes, it is also expected that the interdiffused (DF)-MQW structure can serve as a high-performance FP modulator (vertical cavity) by improving the change of reflectance, $\Delta R$, and the relative reflectance change, $\Delta R / R$, (otherwise known as the fraction of reflectance change) as well as reducing its residual loss in the OFF-state and power consumption. Since only a simple thermal annealing is required for modifying the MQW structure, it becomes a very attractive technology to produce a FP modulator which can tune to operate at a precise operation wavelength $\lambda_{\mathrm{op}}$. So the DF-MQW FP-modulator is expected to become a significant candidate in the vertical optoelectronicsintegration technology. Together with the development of coplanar monolithic integration, a three-dimensional (3-D) optoelectronics integration circuit may become a reality in the near future. On the other hand, for the longitudinal type of waveguide-modulator, the electrooptic effect of DF-MQW's can provide a larger variation in $\Delta n_{\max }$ with a smaller field while a stronger and an almost constant $\Delta n_{\max }$ with a larger field. The phase-modulation properties due to these effects in the DF-MQW's suggest some types of multiwavelength application can be realized by means of fabricating planar integrated multisection waveguide-type modulators and detectors on a single substrate through the technique of selective-area interdiffusion. However, care must be taken in the DF-MQW design, such to choose the best interdiffusion length and electric field. In fact, the DF-MQW can provide large electroabsorption and less chirping in certain selected ranges of wavelengths. These make the DF-MQW's become competitive as an electro-absorptive modulator. In addition, the performance of these DF-MQW devices may be further improved by optimizing the DF-MQW structure together with the use of strain-layered MQW materials. The above attraction stimulates the present study since the precise electronic and optical properties of the DF-MQW must be known before good performing devices can be realized.

In this paper, we report on the comprehensive modeling and detail analysis of the effect of interdiffusion on AlGaAs-GaAs MQW subband-edge eigenstates, including wellto-well coupling and the effect of cladding layers. The effect of interdiffusion on the modified property of the eigenstate is important for optical transitions of the interdiffused MQW, such as interband absorption, which are also presented here. In this work, all of the confined eigenstates of $\mathrm{Al}_{0.3} \mathrm{Ga}_{0.7} \mathrm{As}-$ - GaAs interdiffused MQW are calculated at room temperature for the structure comprising an intrinsic MQW core region, which consists of 10 wells and nine barriers (each with a 100 $\AA$ as - grown width) clad between infinitely thick $\mathrm{Al}_{0.3} \mathrm{Ga}_{0.7} \mathrm{As}$ layer for different extents of the interdiffusion process. This type of structure is chosen because it is typical of the MQW structures currently used in waveguides and modulators. However, the interdiffusion effect on the eigenstate properties of the AlGaAs-GaAs MQW analyzed here should be applicable in general to other unstrained material systems.

The paper is arranged as follows. The interdiffusion model for the MQW is presented in Section II together with the band-edge calculation and valence band-mixing scheme. The dielectric-function approach is used here for the calculation of the polarized absorption coefficients. This is followed by presentation of numerical results in Section III which are generated for a specific as-grown MQW structure as a function of the extent of interdiffusion. A detailed analysis of the confinement profile, eigenstates, and their absorption coefficients is also included. This is followed by a quantitative estimate of how modulator performance changes with the amount of interdiffusion. Finally, conclusions are presented.

\section{MODEL FORMULATION}

In this work, the interdiffusion process to be considered, is based on post-growth thermal-induced interdiffusion of the two excess alloy species across all the heterointerfaces in the MQW structure under stoichiometric conditions, i.e., the $\mathrm{Ga}$ and $\mathrm{Al}$ atoms in the $\mathrm{GaAs}$ well and $\mathrm{AlGaAs}$ barrier layers, respectively, in the AlGaAs-GaAs MQW. The interdiffusion is modeled here using Fick's law with an equal and constant interdiffusion coefficient for the two interdiffusing species [25]. The extent of the interdiffusion is characterized by an interdiffusion length $L_{d}=(D t)^{1 / 2}$, where $D$ and $t$ are the interdiffusion coefficient and time, respectively. ${ }^{3}$ The square of

\footnotetext{
${ }^{3}$ It should be noted here that the definition of $L_{d}$ varies in the literature;
} for instance, some authors define $L_{d}$ as $2(D t)^{1 / 2}$ [9], i.e., $L_{d}^{2}=2$ variance 


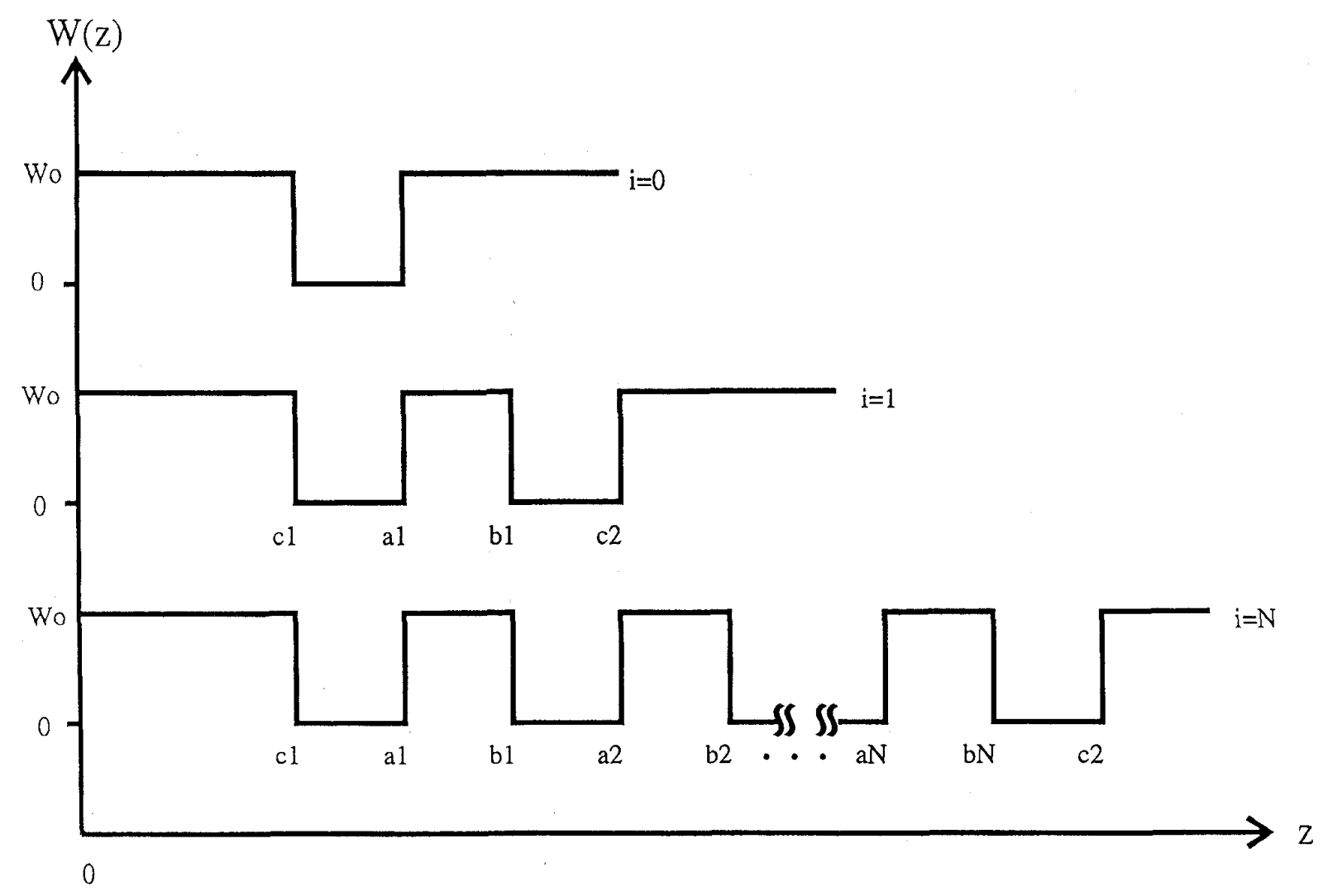

Fig. 1. Schematic illustration of the coordinates used in (1) for the MQW structures.

this length represents half of the variance of the interdiffusion distribution in a linear flow situation (i.e., $L_{d}^{2}=\frac{1}{2}$ variance, and $L_{d}=$ standard deviation $/ \sqrt{2}$ ). The superposition principle is assumed for all of the layers considered in the structure, which includes the MQW core layers and the infinitely thick cladding layers. This is a useful and realistic model for the interdiffusion process in the AlGaAs-GaAs material system. The effects of impurities are not considered here specifically for simplicity, although they are known to enhance the rate of interdiffusion and hence produce an effectively larger $L_{d}$ for the same annealing time. However, since the purpose of the present study is to determine the effects of interdiffusioninduced nonsquare confinement profile, with respect to a different $L_{d}$, on the properties of MQW structures, this approach is adequate. The $\mathrm{Al}$ composition profile $w(z)$ as a function of $L_{d}$ is given by

$$
\begin{aligned}
w(z)= & \frac{w_{0}}{2}\left\{2-\operatorname{erf}\left(\frac{z-c_{1}}{2 L_{d}}\right)+\operatorname{erf}\left(\frac{z-c_{2}}{2 L_{d}}\right)\right. \\
& \left.+\sum_{i=1}^{N}\left[\operatorname{erf}\left(\frac{z-a_{i}}{2 L_{d}}\right)-\operatorname{erf}\left(\frac{z-b_{i}}{2 L_{d}}\right)\right]\right\}
\end{aligned}
$$

where $w_{0}$ is the as-grown $\mathrm{Al}$ concentration in the barrier and the cladding layer, $N$ is the number of barriers within the MQW core of the as-grown structure, $\operatorname{erf}(y)$ denotes the error function, $z \geq 0$ is the growth axis of the MQW layers where and $L_{d}=\sqrt{2}$ standard deviations. the MQW structure is positioned on the positive side, $a_{i}$ and $b_{i}$ are the left and right interface positions, respectively, of the $i$ th as-grown barrier within the MQW core, while $c_{1}$ and $c_{2}$ are that at the two end positions of the as-grown MQW core (i.e., interface between the cladding and core layers), which are shown in Fig. 1. The advantage of using the expression in (1) to describe the interdiffusion-induced nonsquare profile is its ability to model different MQW structures with any number of periods, different widths and heights of the well and barrier layers, as well as different cladding layers, by adjusting the terms involving $c_{1}$ and $c_{2}$. In (1), the omitted $i=0$ case represents a single QW with thick AlGaAs cladding layers, $i=$ odd (even) represents an even (odd) number of wells in the MQW core with an $\mathrm{Al}$ concentration of $w_{0}$ in the cladding layer, as shown in Fig. 1. For cases which use GaAs cladding layers, such as the resonant-tunneling structures, (1) can still be used when the constant has a value of 2 , when terms consist of $c_{1}$ and $c_{2}$ are dropped, and take $N \geq 2$; although this versatility is also demonstrated in a Green's function model [26], (1) is an analytical expression and no numerical integration is necessary. The MQW confinement profile is obtained from $w(z)$ by a method similar to the case of a single QW [10] and is briefly described here.

The diffused Al composition profile defined by (1) is used to obtain the interdiffused QW parameters including bandgap, $E_{g}(z)=E_{g}(w(z))$, and well depth, $\Delta E_{r}\left(L_{d}\right)=$ $Q_{r}\left[E_{g}\left(z_{1}\right)-E_{g}\left(z_{2}\right)\right]$, where the subscript $r$ denotes either the electron in the conduction band $(C)$ or heavy or light holes 


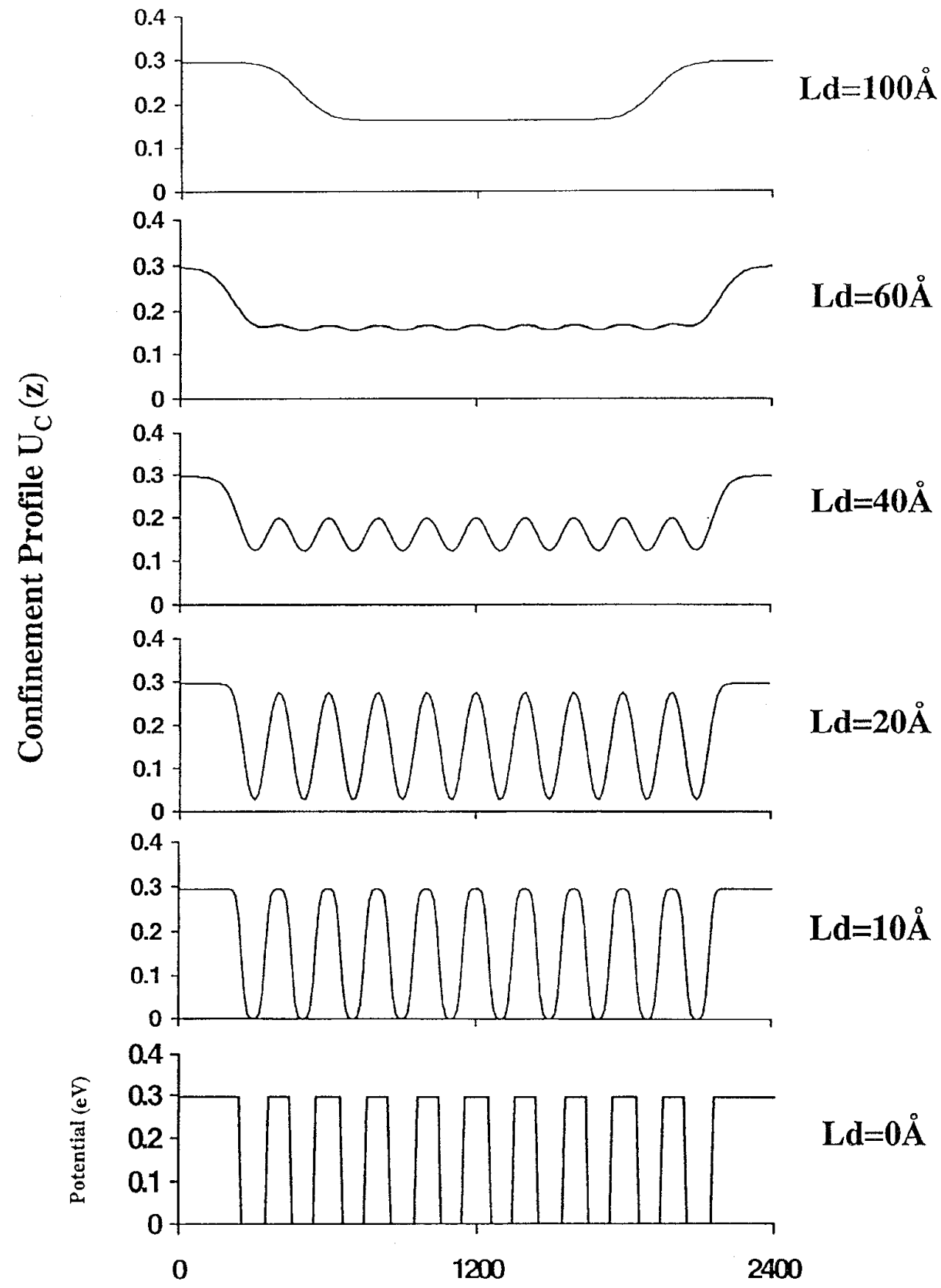

Growth Axis (

Fig. 2. The electron confinement potential for the AlGaAs-GaAs MQW ( $w_{0}=0.3, L_{z}=L_{b}=100 \AA$ ) with several values of $L_{d}$.

in the valence band $(V=H$ or $L), Q_{r}$ is the band offset splitting, and $z_{1}$ and $z_{2}$ refer to the positions corresponding to top of the barrier and bottom of the well, respectively. The interdiffused MQW confinement profile, $U_{r}(z)$, is defined as $U_{r}(z)=Q_{r}\left[E_{g}(w(z))-E_{g}(w=0)\right]$. The convention here is to define zero potential to be both at the bottom of the as-grown square MQW and positively up in both bands.

The QW subband edge at the zone-center of $\Gamma_{6}$-valley symmetry can be calculated separately for electrons and holes, using the Ben-Daniel and Duke model [27]. A $z$-position dependent effective mass determined by the interdiffused composition profile is used in the one-dimensional Schrödingerlike equation, for the envelope function $\psi_{r} \ell(z)$, which is written as

$$
-\frac{\hbar^{2}}{2} \frac{d}{d z}\left[\frac{1}{m_{\perp r}^{*}(z)} \frac{d \psi_{r \ell}(z)}{d z}\right]+U_{r}(z) \psi_{r \ell}(z)=E_{r \ell} \psi_{r \ell}(z)
$$

where $\ell=1,2, \cdots$ are the pure MQW subband levels for either the electrons $(C \ell)$ or holes $(V \ell)$, respectively; these 


\section{DF-MQW $(\mathrm{Al}=0.3, \mathrm{Lz}=100 \mathrm{~A}, \mathrm{Lb}=100 \mathrm{~A})$}

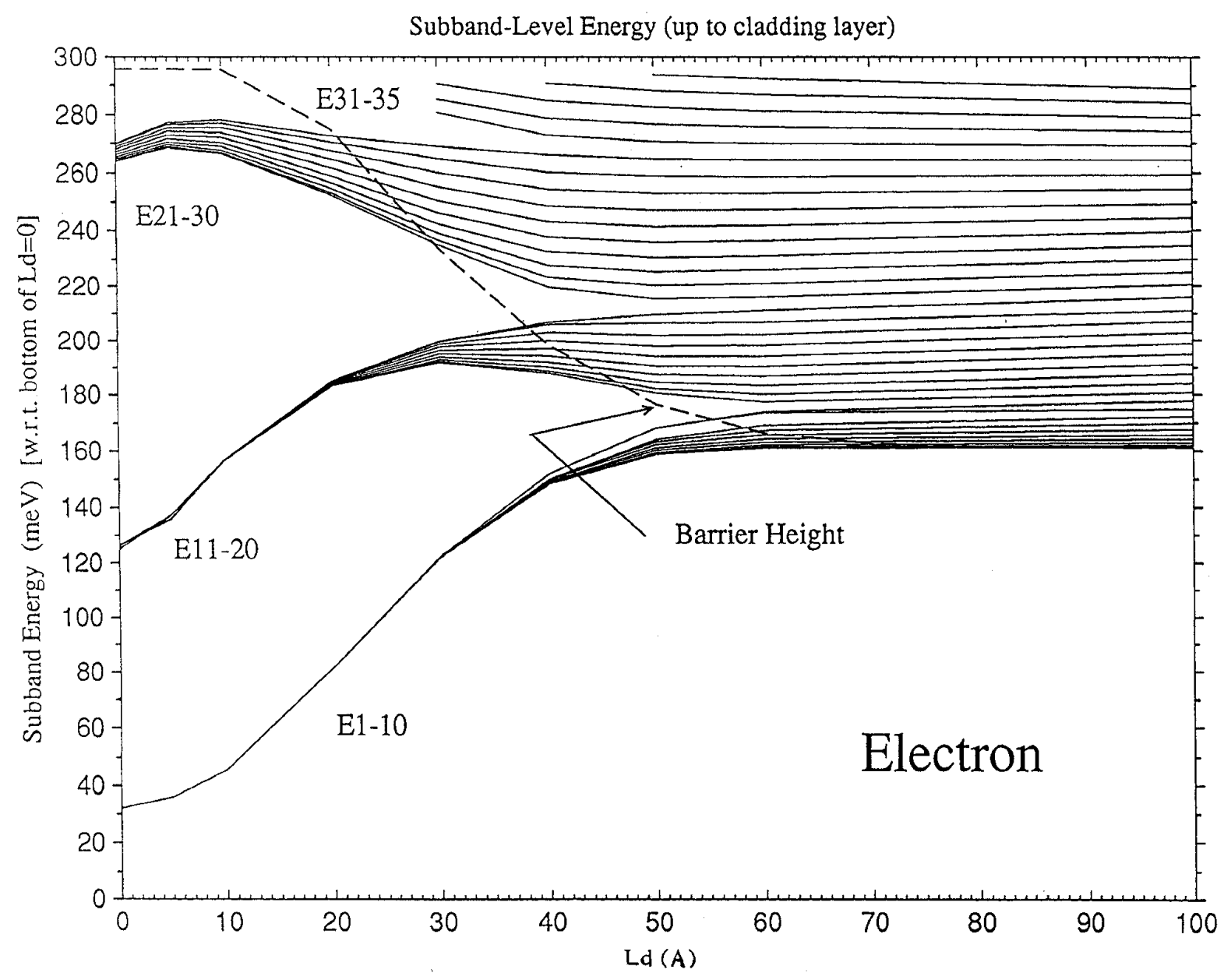

(a)

Fig. 3. The subband-edge eigenstate energy of the DF-MQW ( $w_{0}=0.3, L_{z}=L_{b}=100 \AA$ ) as a function of $L_{d}$ for (a) electron, (b) heavy hole, and (c) light hole. The dashed line indicates the height of barriers in the MQW core with zero taken from the bottom of the as-grown $\left(L_{d}=0\right)$ well. The energy levels are arranged in total number counting from the lowest level.

pure sublevels $(\ell)$ are due to the confinement effect only, where well-to-well coupling is ignored, i.e., as if they are single wells; $m_{\perp r}^{*}(w(z))$ is the carrier effective mass in the $z$ direction which is perpendicular to the QW layers; $E_{r \ell}$ is the subband-edge energy, and the zero energy is taken to be at the bottom of the as-grown MQW. The eigenvalue problem, (2), is solved numerically for the entire MQW structure using a finite-difference method with the boundary conditions taken to be zero at the end of a cladding layer of finite thickness, i.e., $\psi_{r \ell}(0)=\psi_{r \ell}\left(L_{f}\right)=0$, where $L_{f}=N L_{b}+(N+1) L_{z}+$ $2 L_{c}\left(L_{c}\right.$ is the cladding layer width), i.e., the MQW core and cladding are embedded in a well of width $L_{f}$ with an infinitely high barrier to represent the infinitely thick cladding layer. This method permits all the subband eigenstates to be determined, including those due to well-to-well coupling.

The other standard methods of calculation for the MQW eigenstates are tight-binding [32] and transmission tunneling [23]. The former method takes into account the pre-assumption of some well-to-well coupling with an approximate coupling strength of the ground states which introduces inaccuracy in cases of strong coupling. It also ignores coupling of the upper states. The latter model becomes inaccurate in thick multilayered structures and inapplicable in the case of thick barriers.

In order to calculate the absorption coefficient accurately, the nonparabolic band-structure dispersion along the transverse $x-y$ direction of the QW structure is required. The valencesubband dispersion for the mixing between heavy- and lighthole states (denoted by $V=H$ and $V=L$, respectively) are considered here for the transverse (parallel to QW layers) wave vector $\left\|\boldsymbol{k}_{x y}\right\|=k_{\|}=0$, which can be solved in the neighborhood of a high-symmetry zone-center (i.e., $k \|=$ 0 ) using approximation methods such as the one developed previously using an effective Hamiltonian [28]. This approach has been applied in single DFQW structures [15] and is based on the analogous application of the $k \cdot p$ method to the subbands. The mixed envelope function in the valenceband, $\Psi_{V}\left(k_{t}, z\right)$, at any finite $k_{t}$ not too far away from the 


\section{DF-MQW (Al=0.3, Lz=100A, Lb=100A)}

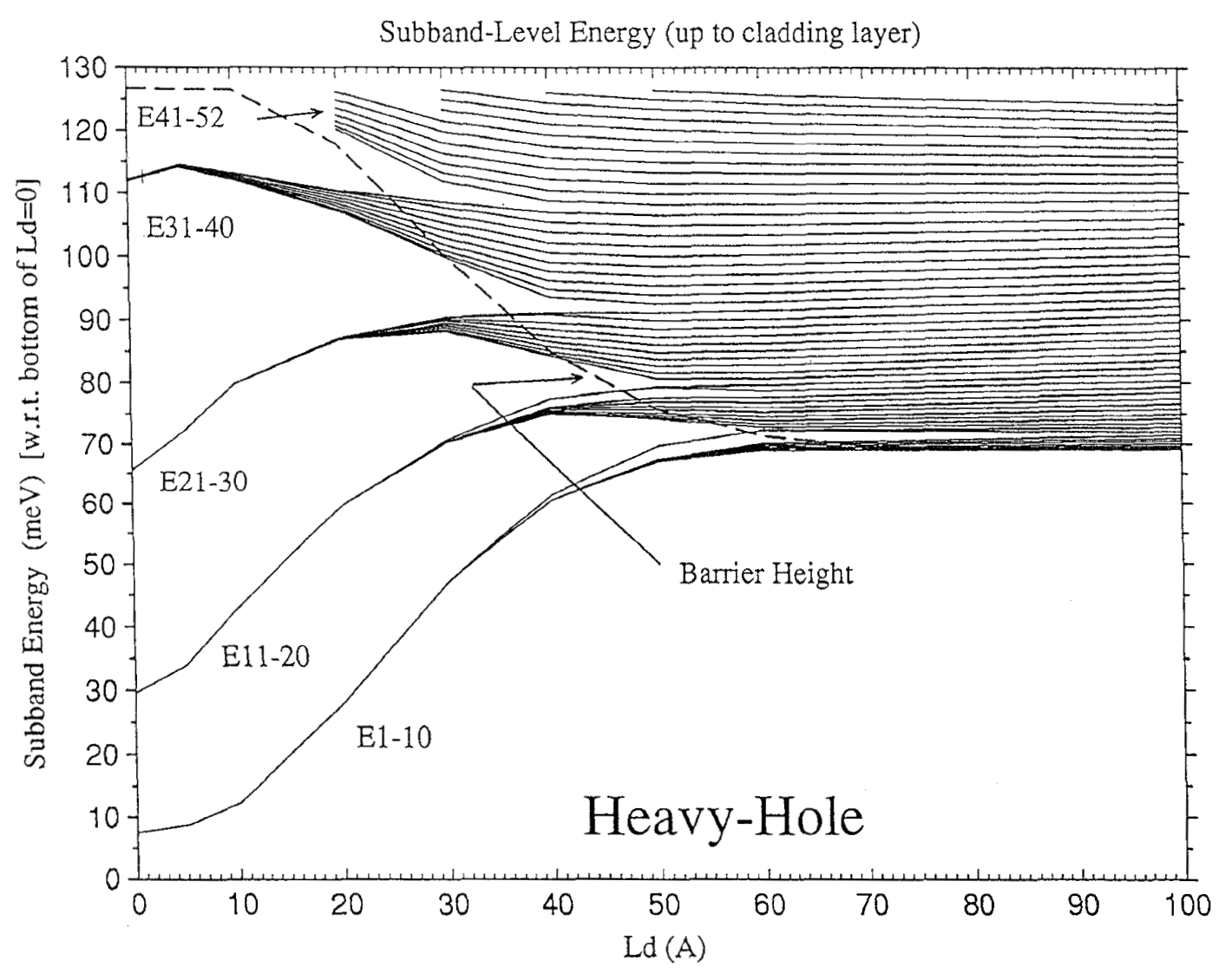

(b)

Fig. 3. (Continued.) The subband-edge eigenstate energy of the DF-MQW ( $w_{0}=0.3, L_{z}=L_{b}=100 \AA$ ) as a function of $L_{d}$ for (b) heavy hole. The dashed line indicates the height of barriers in the MQW core with zero taken from the bottom of the as-grown $\left(L_{d}=0\right)$ well. The energy levels are arranged in total number counting from the lowest level.

highest symmetry point $\left(k_{\|}=0\right)$ can be expressed as a linear combination of the degenerate envelope functions $\psi_{V \ell}(z)$ without mixing at $k_{\|}=0$, which are obtained a priori from the solution of (2). The validity of the approximation is dependent upon a limited range of $k_{\|}$, since only a finite-dimension basis set for the expansion is used in practice. However, a small range of $k_{\|}$near 0 is sufficient for the interband transitions around the absorption edge, where the QW effects are most significant. The basis for the expansion is defined as

$$
\Psi\left(k_{\|}, z\right)=\sum_{\ell=1}^{N} \sum_{V=H, L} d_{V, \ell}\left(k_{\|}\right) \psi_{V, \ell}(z)
$$

where $d$ is the expansion coefficient, $V$ includes the lifted fourfold degenerate-hole states, and $N$ is the maximum number of bases taken in each $V$, which includes all subbands. The effective Hamiltonian is

$$
\left[\begin{array}{cccc}
E_{+H} & C & B & 0 \\
C^{*} & E_{-L} & 0 & B^{T} \\
B^{*} & 0 & E_{+L} & C^{T} \\
0 & B^{*} & C^{*} & E_{-H}
\end{array}\right]
$$

where

$$
\begin{aligned}
B_{\ell \ell^{\prime}} & =\sqrt{3} \frac{\hbar^{2}}{m_{0}} \gamma_{2}\left(-k_{x}-i k_{y}\right) \int_{0}^{L_{f}} d z-\psi_{H \ell}(z) \frac{\partial}{\partial z} \psi_{L \ell^{\prime}}(z) \\
C_{\ell \ell^{\prime}} & =\frac{\sqrt{3}}{2} \frac{\hbar^{2}}{m_{0}} \gamma_{2}\left(k_{x}-i k_{y}\right)^{2} \int_{0}^{L_{f}} d z-\psi_{H \ell}(z) \psi_{L \ell^{\prime}}(z) \\
E_{ \pm H} & =\left[E_{ \pm H \ell}-\frac{\hbar^{2}}{2 m_{\| H}} k_{\|}^{2}\right] \delta_{\ell \ell^{\prime}} \\
E_{ \pm L} & =\left[E_{ \pm L \ell}-\frac{\hbar^{2}}{2 m_{\| H}} k_{\|}^{2}\right] \delta_{\ell \ell^{\prime}}
\end{aligned}
$$

where $\gamma_{2}(\omega)$ is the Lüttinger parameter, the sign $\pm H, L$ stands for spins, $\ell$ and $\ell^{\prime}$ also represent an index for different entry in the matrix, $\delta$ denotes the delta function at $\ell-$ $\ell^{\prime}$ which represents the diagonal terms, and $L_{f}$ is defined earlier. The effect of the introduction of this larger well is to determine numerically the continuum levels above the top of the DF-MQW. It should be noted that, in the absence of an applied electric field or any other mechanism that will distort the inversion symmetry assumed here, the symmetric MQW confinement-potential profile causes the valence subband to exhibit a twofold Kramer's spin degeneracy. 


\section{DF-MQW $(\mathrm{Al}=0.3, \mathrm{Lz}=100 \mathrm{~A}, \mathrm{Lb}=100 \mathrm{~A})$}

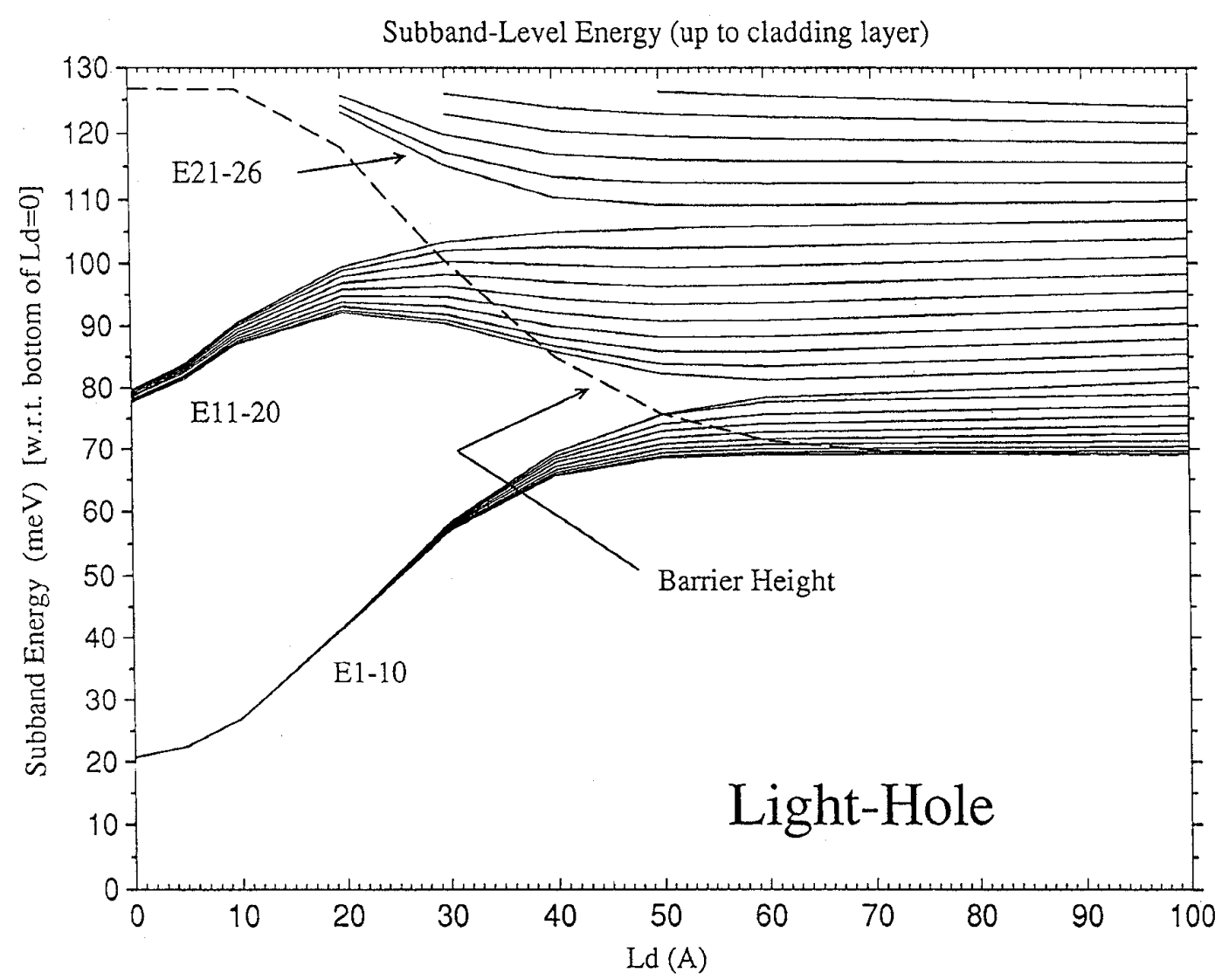

(c)

Fig. 3. (Continued.) The subband-edge eigenstate energy of the DF-MQW ( $w_{0}=0.3, L_{z}=L_{b}=100 \AA$ ) as a function of $L_{d}$ for (c) light hole. The dashed line indicates the height of barriers in the MQW core with zero taken from the bottom of the as-grown $\left(L_{d}=0\right)$ well. The energy levels are arranged in total number counting from the lowest level.

The interband transitions are considered here without the electron-hole coulomb interaction. The omission of this excitonic effect can introduce inaccuracy at the band-edge in certain QW cases, such as those of an undoped structure, of high-purity material and operation at low temperatures. Thus, the present formulation demonstrates a somewhat simplified absorption-coefficient calculation without the exciton resonance at the absorption edge which may be unresolved even after interdiffusion. However, these bare transition states are the long-range exciton-continuum states and serve as a background to the absorption coefficient. It should be noted that in the case of the optical properties of an MQW laser structure, where excitons are not important, the present formulation of the imaginary part of the dielectric function is of importance.

Once the electron and hole-envelope wavefunctions and subband energy levels of the DF-MQW structure have been determined, the linear absorption coefficient $\alpha(\omega)$ may be determined in terms of the imaginary part of the dielectric function $\varepsilon_{2}(\omega)$ using the relation [29]

$$
\alpha(\omega)=\frac{\omega \varepsilon_{2}(\omega)}{c_{0} n_{R}(\omega)}
$$

where $n_{R}(\omega)$ and $c_{0}$ are the refractive index of the QW and the velocity of light in vacuo, respectively. The incident electromagnetic radiation is assumed to propagate parallel to the $x-y$ plane (perpendicular to $z$ axis) of the QW layer, therefore $\alpha$ and $\varepsilon_{2}$ are anisotropic with polarization (TE and TM) [33]. Since we are interested in the QW quantization effect which manifests itself over a limited energy range above the bandgap, we use a $\varepsilon_{2}(\omega)$ calculation, which is based on the direct interband transitions around the absorption edge in the $\Gamma$ valley and an additional factor to take account of the polarization. $\varepsilon_{2}$ is expressed as

$$
\begin{aligned}
\varepsilon_{2}^{\mathrm{TE}, \mathrm{TM}}(\omega)= & \left.\frac{e^{2} M_{b}^{2} \wp}{\pi \varepsilon_{0} m_{0}^{2} \omega^{2} L_{z}} \sum_{\ell, \ell^{\prime}} \int d k\left|\left\langle\psi_{C \ell}\right| \psi_{V \ell^{\prime}}\right)\right|^{2} \\
& \cdot \mathcal{L}\left(E_{C \ell}\left(k_{\|}\right)-E_{V \ell^{\prime}}\left(k_{\|}\right)-\hbar \omega\right)
\end{aligned}
$$

where $\varepsilon_{0}$ is the permittivity of free space, $e$ and $m_{0}$ are the electron charge and rest mass, respectively, $M_{b}^{2}=m_{0}^{2} P^{2} / 3 \hbar$ and $P$ are given by Kane's model, $\mathcal{L}$ is the Lorentzian broadening factor with half-width-half-maximum $\Gamma_{b}$, the summation in (6) is over all the subband and minisubband bound and continuum states in both bands, and $\wp$ is the polarization 


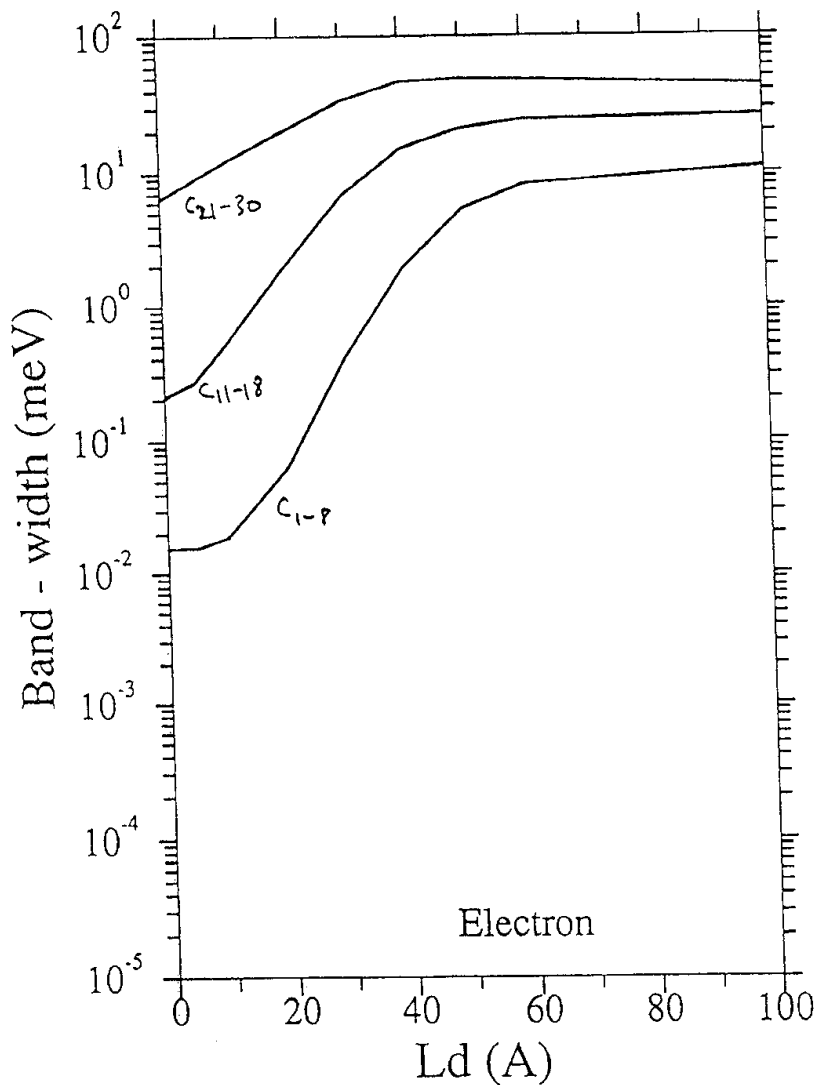

(a)

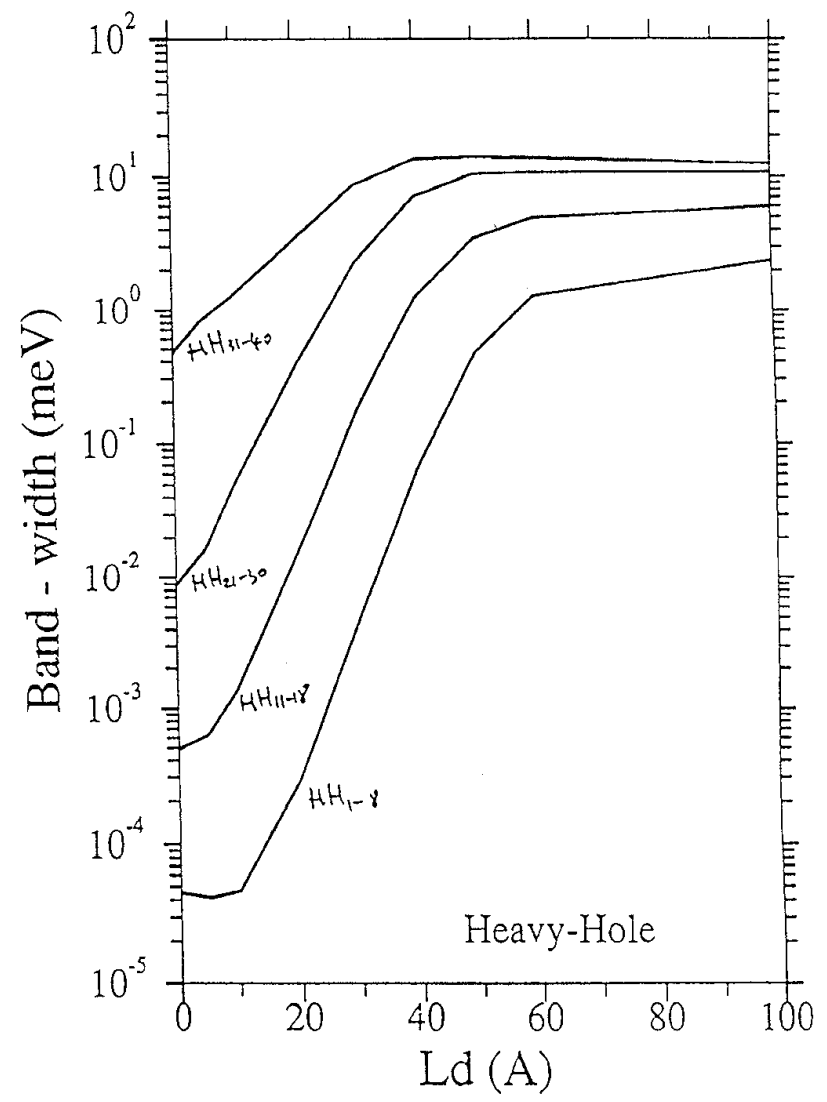

(b)

Fig. 4. Band-width of the minisubbands in $\log$ scale as a function of $L_{d}$ for (a) electron and (b) heavy-hole.

TABLE I

Material Parameter for AlGaAs-GaAs QW

\begin{tabular}{|c|c|c|c|}
\hline & $\mathrm{AI}_{\mathrm{w}} \mathrm{Ga}_{1-\mathrm{w}} \mathrm{As} / \mathrm{GaAs}$ & Units & Ref. \\
\hline $\mathrm{Q}_{\mathrm{C}}: \mathrm{Q}_{\mathrm{V}}$ & $0.7: 0.3$ & -- & 38 \\
$\mathrm{E}_{\mathrm{g}}(\mathrm{w})$ & $1.424+1.594 \mathrm{w}+\mathrm{w}(1-\mathrm{w})(0.127-1.31 \mathrm{w})$ & $\mathrm{eV}$ & 39 \\
$\Delta_{0}(\mathrm{w})$ & $0.34-0.04 \mathrm{w}$ & $\mathrm{eV}$ & 40 \\
$\gamma_{\mathrm{z}}(\mathrm{w})$ & $2.02-1.12 \mathrm{w}$ & $\mathrm{m}_{0}$ & 41 \\
$\mathrm{~m}^{*} \mathrm{c}(\mathrm{w})$ & $0.0665+0.0835 \mathrm{w}$ & $\mathrm{m}_{0}$ & 41 \\
$\mathrm{~m}^{*}{ }^{\perp \mathrm{H}}(\mathrm{w})$ & $0.33+0.18 \mathrm{w}$ & $\mathrm{m}_{0}$ & 41 \\
$\mathrm{~m}^{*}{ }^{\perp \mathrm{L}}(\mathrm{w})$ & $0.09+0.09 \mathrm{w}$ & $\mathrm{m}_{0}$ & 41 \\
$\mathrm{~m}^{*} \mid \mathrm{H}(\mathrm{w})$ & $0.11+0.10 \mathrm{w}$ & $\mathrm{m}_{0}$ & 41 \\
$\mathrm{~m}^{*} \mid \mathrm{L}(\mathrm{w})$ & $0.20+0.15 \mathrm{w}$ & $\mathrm{m}_{0}$ & 41 \\
$\Gamma_{\mathrm{b}}$ & 5 & $\mathrm{meV}$ & 42 \\
\hline
\end{tabular}

$m_{0}$ and $\epsilon_{0}$ are the electron mass and static dielectric constant, respectively, in free space.

factor at the band-edge where $\wp^{\mathrm{TE}}=3 / 2(H H), 1 / 2(L H)$ and $\wp^{\mathrm{TM}}=0(H H), 2(L H)$.

\section{Results AND Discussion}

The MQW structure analyzed here consisted of 10 wells and nine barriers in the core region of $\mathrm{Al}_{w} \mathrm{Ga}_{1-w} \mathrm{As}-$ - GaAs materials system and two finite cladding layers of $\mathrm{Al}_{w} \mathrm{Ga}_{1-w} \mathrm{As}$, both of the same $\mathrm{Al}$ concentration, where the as-grown structural parameters are $w=w_{0}=0.3, L_{z}=100$ $\AA, L_{b}=100 \AA$, and $L_{c}=600 \AA$ (thus $L_{f}=3100 \AA$ ), and the other material parameters are given in Table I. The discussion below is based on this structure unless otherwise specified. In this study, the effect of interdiffusion on the MQW is based on the modification of the confinement profile, eigen-energy, eigen-wavefunctions, well-coupling band-width, and interband optical transitions by varying the interdiffusion length $L_{d}$.

\section{A. Confinement Profile}

The electron-confinement profile is shown in Fig. 2 for several values of $L_{d}$. It can be seen that $U_{C}(z)$ changes gradually from an as-grown square $\left(L_{d}=0\right)$ to a nonlinear shape initially $\left(0 \leq L_{d}<20 \AA\right.$ ) while retaining the magnitude 


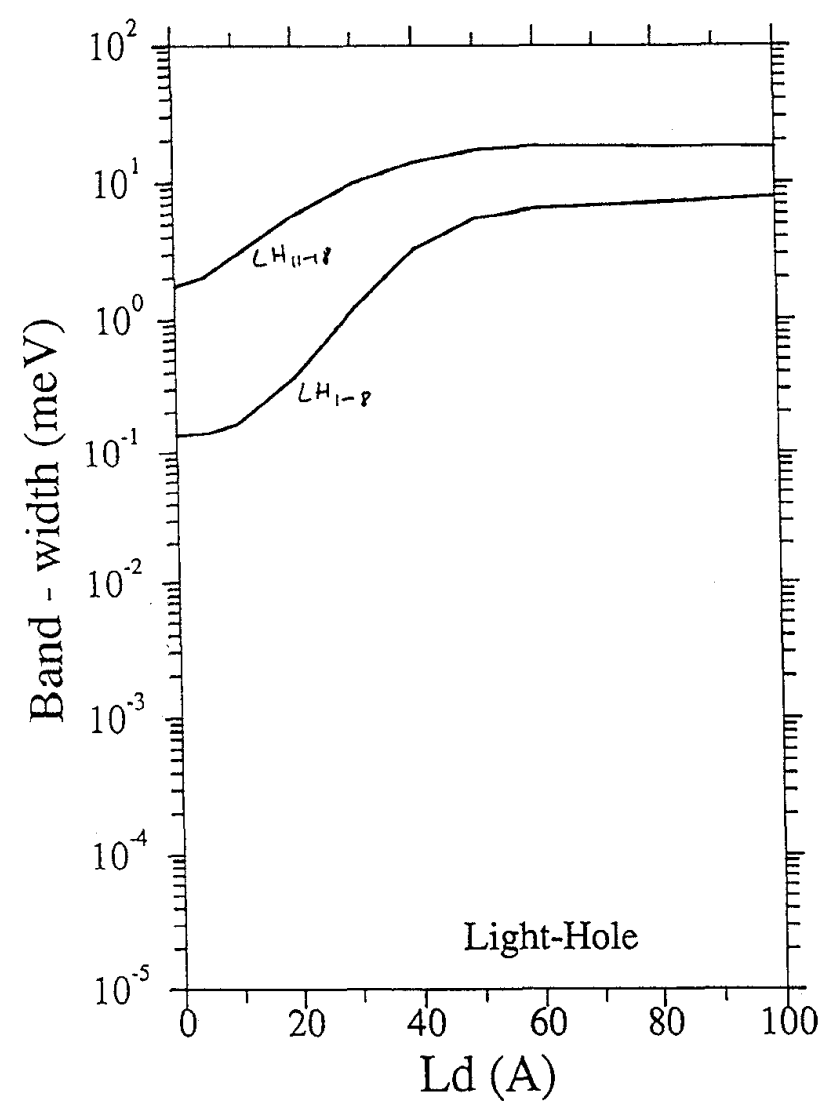

(c)

Fig. 4. (Continued.) Band-width of the minisubbands in log scale as a function of $L_{d}$ for (c) light-hole.

of its confinement depth $\Delta E_{C}\left(L_{d}\right)$, and as $L_{d} \geq 20 \AA$, the QW depth starts to reduce and becomes zero at $L_{d}=100 \AA$. The mid-point of the confinement depth (potential at $\frac{1}{2} \Delta E_{C}$ ), which represents the average of the well and barrier potential due to interdiffusion, gradually moves toward a potential which corresponds to the average Al composition between the well and barrier, i.e., $w=0.15$. After extensive interdiffusion between the well and barrier (i.e., $L_{d}=100 \AA$ here) their $\mathrm{Al}$ content should be averaged out (i.e., $\frac{1}{2}(0+0.3)=0.15$ in our case). However, it should be noted that due to "bowing," the corresponding potential in the core region is not the simple average between the well and the barrier potentials (i.e., $\left.U_{C}\left(L_{d}=100 \AA\right) \neq \frac{1}{2} U_{C}\left(L_{d}=0\right)=147.819 \mathrm{meV}\right)$. In fact, this average potential is $U_{C}=0.7\left[E_{g}(w=0.5)-E_{g}(0)\right]=$ $161.1671 \mathrm{meV}$ (see Fig. 2). The analysis for the hole is similar to that of the above with $U_{V}\left(L_{d}=100 \AA\right)=69.073$ $\mathrm{meV}$ and $\frac{1}{2} U_{V}\left(L_{d}=0\right)=63.351 \mathrm{meV}$. The confinement profile now becomes that of a wide single $\mathrm{QW}$ with gradedinterfaces and with the two cladding layers, which act as the only barriers, and the entire core region acts as the well. This is the intermediate stage of interdiffusion for a MQW structure with nonzero cladding height (such as AlGaAs) and for which bandgap remains stable (constant $E_{g}$ ) within this range, especially in the central core region. Beyond $L_{d}=$ $100 \AA$, the interdiffusion results in a graded profile for the $\mathrm{Al}$ composition (nonconstant $E_{g}$ ) across the entire core region until the thick cladding layers start to affect the complete composition profile. Eventually, $w(z)$ approaches an average constant value between the initially averaged core composition and that of the cladding layers, if it has a finite thickness. On the other hand, in the case of an infinite-thickness cladding layer, the core $\mathrm{Al}$ composition will approach that of the asgrown cladding layer as $L_{d} \rightarrow \infty$.

\section{B. Subband-Edge Eigenstates}

In order to discuss the eigenstate results of the interdiffused MQW structure considered here, it will be helpful to define an additional system of labeling. The eigenstates of a single QW are in fact the so-called edge (minima) of the subbands, which arise due to the splitting of the bulk bands caused by quantum confinement. These eigenstates are labeled by an index $\ell=1,2, \cdots$ to denote their corresponding energy levels and wavefunctions, see (2). However, when $N_{w}$ identical wells are constructed in a MQW structure, each eigenstate of the individual well that belongs to the same subband are coupled together to form a mini-band of their own. The states within these mini-bands are now denoted by a second index $\xi=1,2, \cdots, N_{w}$, so that the energy and wavefunction of an eigenstate are written as $E_{r \ell, \xi}$ and $\psi_{r \ell, \xi}$, respectively, where for instance $E_{C 2,4}$ and $\psi_{C 2,4}$ refer to the fourth eigenstate within the mini-band of the second subband. It should be noted that $N_{w}=1$ refers to a single QW, while a degenerate $E_{r \ell, \xi}$ (for all $\xi$ ) is considered to be the case for weak or insignificant well-to-well coupling.

Fig. 3 shows the eigenstate energy for three cases of electron, heavy, and light holes with respect to the bottom of the as-grown QW (i.e., the bulk GaAs band-edge in the present case). These states are grouped in tens due to the mini-band of 10 eigenstates that arise from the $10 \mathrm{QW}$ 's considered here. The energy of the barrier height $U_{b}$, which is also measured with respect to the GaAs edge at the center of the MQW structure, is indicated by a dash-line. It can be seen that as interdiffusion starts $\left(L_{d}<20 \AA\right)$, there are three electron, four heavy-hole, and two light-hole degenerate subbands, where the degeneracy of all the respective top subbands are slightly raised, which is a consequence of the weaker confinement near the top of the well. As interdiffusion proceeds further, the lifting of this degeneracy increases and it turns into a miniband in all three cases, where the higher energy and lighter mass subbands are enhanced more significantly, which is due in general to their weaker confinement. The splitting of the degenerate levels becomes evenly spaced and fully distributed throughout all the subbands in all cases as the interdiffusion proceeds far enough so that the subbands are above the barrier energy $U_{b}$, as indicated in the region above the dash-line. These states can now be considered as bulk-like states, since they are now in a single $\mathrm{QW}$ within an as-grown width of $10 L_{z}+9 L_{b}=1900 \AA$. However, as it can be seen for subbands near and above $U_{b}$. the mini-band nature remains because the states are affected by the slightly modulated MQW structure; it behaves like the transmission-scattering states above a nonflat surface. Also, there are a number of states introduced at the top of the MQW core structure, due to interdiffusion, which 


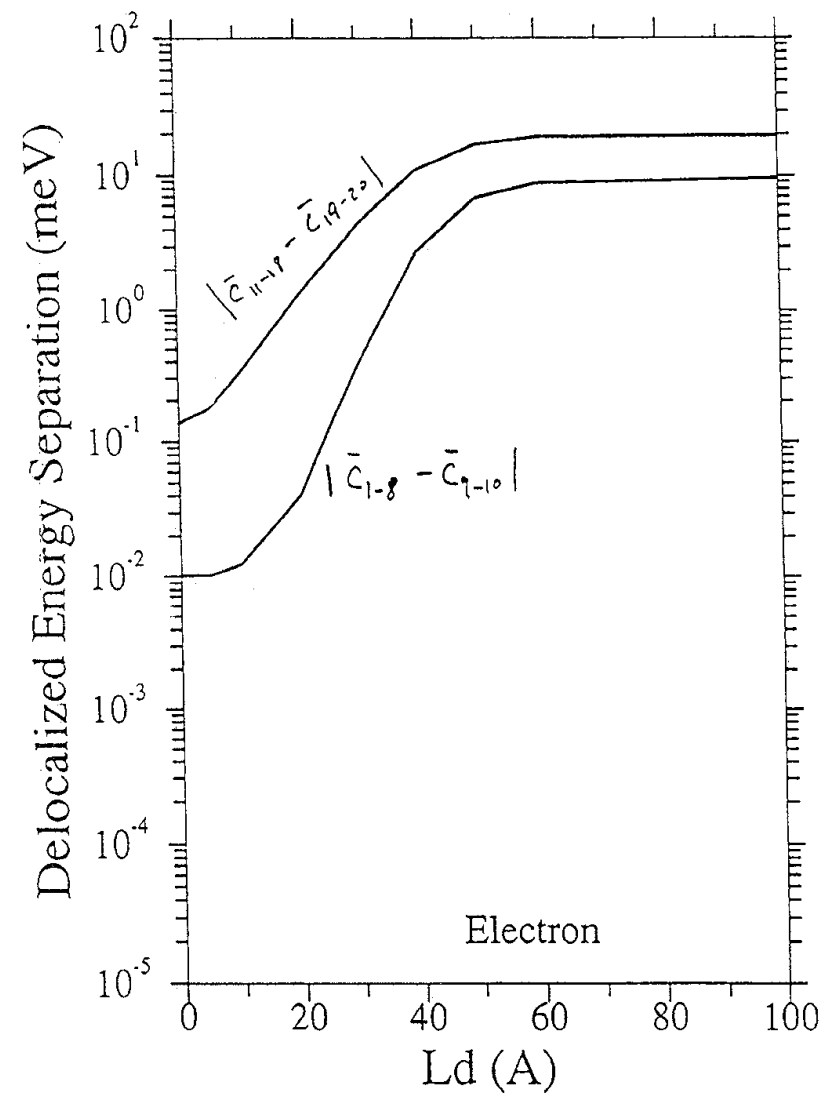

(a)

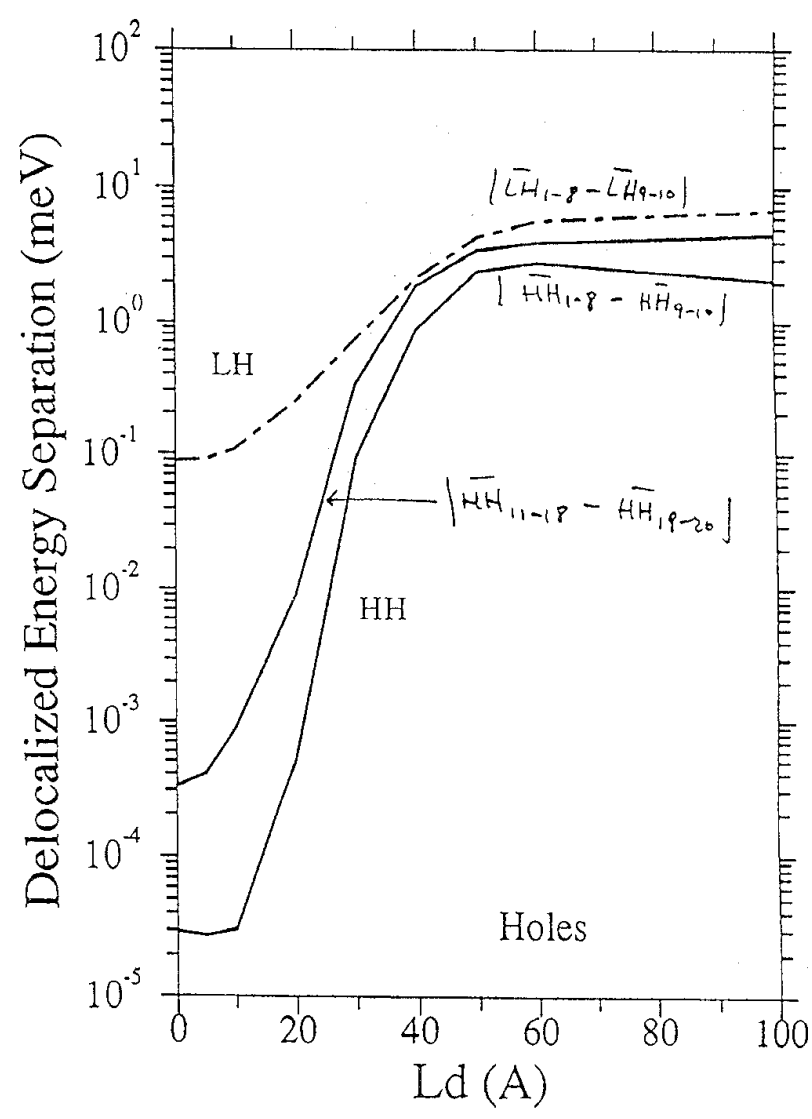

(b)

Fig. 5. The delocalization energy separation between the center MQW core localized wavefunctions and that at the far ends near the cladding layers. (a) Electron and (b) heavy-hole.

are the $C(\ell=4 ; 1 \leq \xi \leq 5), H H(\ell=5 ; 1 \leq \xi \leq 10)$ and $(\ell=6 ; 1 \leq \xi \leq 2)$, and $L H(\ell=3 ; 1 \leq \xi \leq 6)$, and in fact an increasing number of states will be pull down and from the continuum and become confined near the top of the MQW core as $L_{d}$ is increased further until eventually the MQW core merges with the cladding layers and becomes an averaged bulk material.

The lifting of the degeneracy is due to the tunneling of the confined carriers into the neighboring wells and causes an increase of the well-to-well coupling of the eigenstates which cause these levels to split both coherently and evenly into a mini-band. The increase of the coupling strength is a consequence of the interdiffusion which causes the barriers to become lower and narrower, thereby enabling the confined carriers to tunnel easier.

One point to note here is the increase of all the eigenenergies during the early stages of interdiffusion $\left(L_{d}<10\right.$ $\AA$ ), where the depth of the well remains constant, as can be found from the dashed line. This means that the eigen-energy increases with $L_{d}$ within this region of interdiffusion, which can be explained by the narrowing of the well for small $L_{d}$, which is also found in single interdiffused QW's [10], [31]. The reason is that the upper high-energy levels also increase, although the effective well width is wider, which is due to the fact that the confined carrier has a larger probability in the center of the symmetric QW (since $\psi^{2}$ is bell-shaped), so that it experiences more of a narrow well in the center than a wider well near $\pm L_{z} / 2$.

Another point worth noting is the ninth and tenth states in the first minisubband and the nineteenth and twentieth states in the second minisubband appear to group together and split away from their original group of 10 (see Fig. 3). This is due to the antisymmetry of the two wells next to the cladding barrier layer where interdiffusion on the left- and right-hand side interfaces are different, since one is due to a finite source and the other from an infinite-like source. This will become clearer later when their wavefunctions are discussed.

The mini-band width of the electron, heavy-, and lighthole subbands as a function of $L_{d}$ are shown in Fig. 4(a)-(c), respectively. Note that only the first eight states $(1 \leq \xi \leq 8)$ are considered here for the first and second minisubbands $(\ell=$ $1,2)$. It can be seen for all three cases that, as interdiffusion starts, the mini-band width increases quite rapidly until $L_{d}$ reaches $50 \AA$, after which point they slowly level up as $L_{d} \rightarrow 100 \AA$. There are two exceptions, one for the first $\mathrm{HH}$ minisubband $(\ell=1)$ at $L_{d} \approx 5 \AA$, where the width of the band actually decreases slightly, and the other for the third and fourth subbands $(\ell=3,4)$ of the heavy-hole and the third minisubband $(\ell=3)$ for the electron where the band-width increases to a maximum at $L_{d} \approx 50 \AA$ and then decreases slightly. The first of these can be explained by the narrowing of the wells and thus a widening of the barrier near 
$\mathrm{DF}-\mathrm{MQW}(\mathrm{Al}=0.3 ; \mathrm{Lz}=100 \mathrm{~A} ; \mathrm{Lb}=100 \mathrm{~A}) \mathrm{Ld}=10 \mathrm{~A}$ Electron

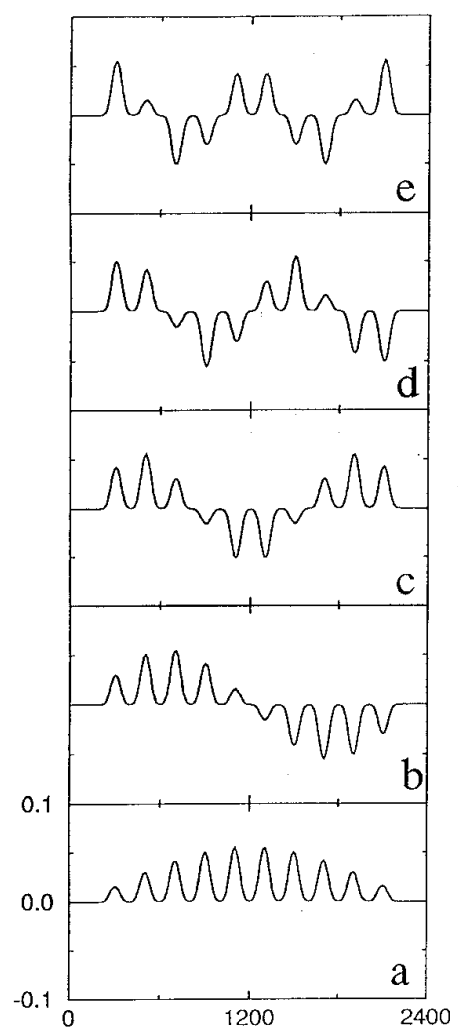

(a)

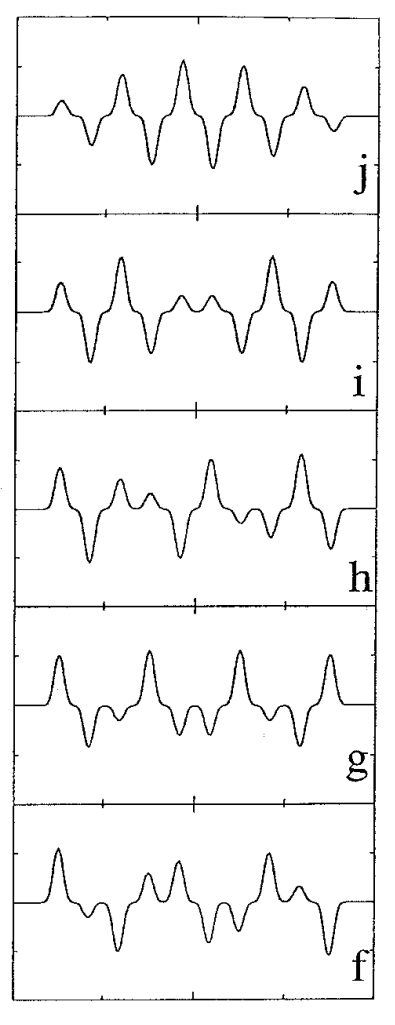

g.

$\int_{-0.1}^{0.1} 0$

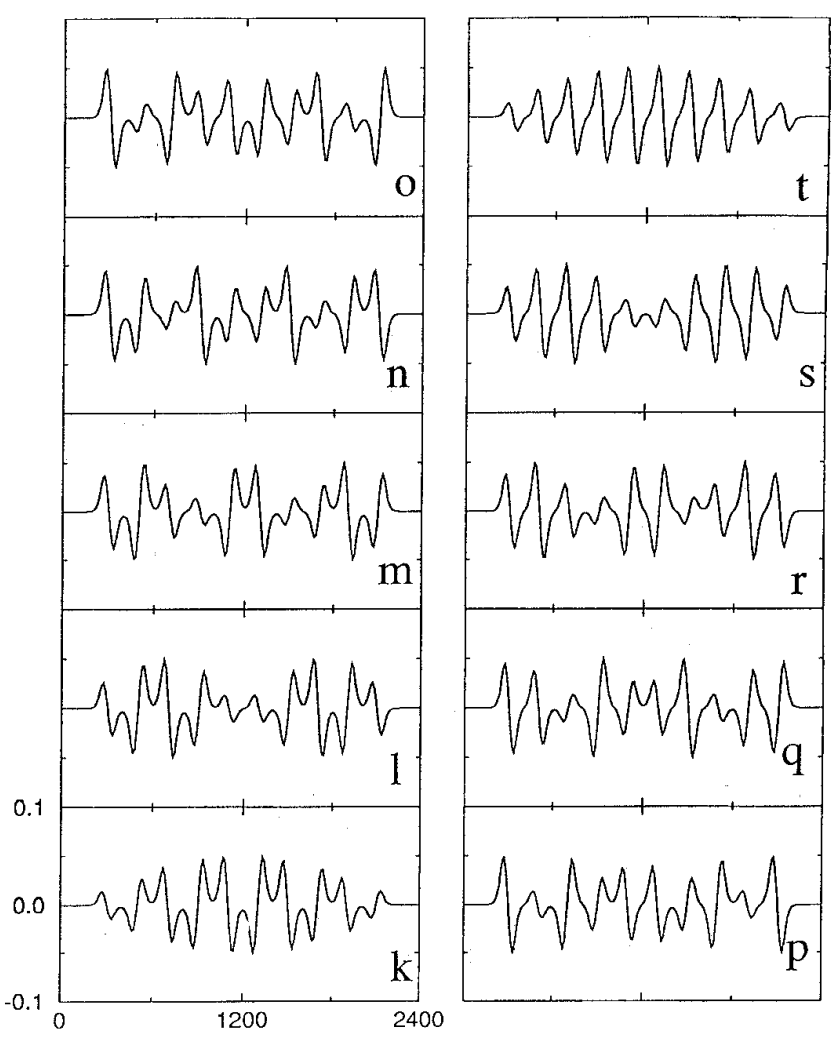

(b)

Ld $=10 \mathrm{~A}$ Electron $11-20$

Fig. 6. The first (lowest energy) 20 eigenstates of electron for three cases where a-j denote the first 10 and $\mathrm{k}-\mathrm{t}$ denote the next $10 . \mathrm{Al}=0.3, \mathrm{Lz}=$ $100 \AA$, and $\mathrm{Lb}=100 \AA$ for $(\mathrm{a})-(\mathrm{b}) \mathrm{Ld}=100 \AA$.

TABLE II

SUBBAND ENERGY (meV)

\begin{tabular}{||c|c|c|c|c|c|}
\hline first mini- & \multicolumn{5}{|c|}{$\mathrm{L}_{\mathrm{d}}(\AA)$} \\
\cline { 2 - 6 } subband states & 0 & 30 & 40 & 50 & 60 \\
\hline HH1 & 7.4292 & 47.220 & 60.593 & 67.014 & 68.825 \\
HH8 & 7.4292 & 47.225 & 60.659 & 67.477 & 70.100 \\
HH9 & 7.4292 & 47.316 & 61.505 & 69.670 & 72.264 \\
HH10 & 7.4292 & 47.316 & 61.505 & 69.670 & 72.305 \\
\hline
\end{tabular}

the bottom where the $\mathrm{HH}(\ell=1)$ level is located, as this would reduce the well-to-well coupling for such eigenstates, therefore reducing the coupling-induced mini-band-width. The second phenomenon for the higher energy levels is due to the inclusion of the last two states $(\ell=3 ; \xi=9,10)$ and $(\ell=4 ; 1 \leq \xi \leq 10)$ which are not considered in the first two subbands $(\ell=1,2)$, as these states will decouple from the rest of the lower energy states within the minisubband, since the QW's shape are no longer identical at large enough $L_{d}$ 's. In fact, if we list the actual first minisubband energies for $H H(\xi=1,8,9,10)$, as shown in Table II, all of the energies start at the same value for $L_{d}=0$ and the energy separation between the first eight states and the last two states increases from zero to approximately $3 \mathrm{meV}$ as $L_{d}$ increases.

The band-widths for the first minisubband are shown in Table III with accuracy up to six decimal places of a meV. For comparison, the tight-binding method of Bastard [32] has also been used to determine this band-width. As can be seen in Table III, the tight-binding method agrees very well with our full numerical calculation for small $L_{d}$ 's except for a slight overestimation of the energy, while for larger $L_{d}$ 's it underestimates the energy by a small amount. However, for the case of $L_{d}=60 \AA$, the tight-binding method underestimates the energy by over $25 \%$ for both cases of the lighter mass carriers, i.e., $C$ and $L H$. This underestimation is due to the strong well-to-well coupling effect and band-mixing which are not fully accounted for in the tight-binding method. In spite of these problems, both methods agree quite well in general.

The delocalization effect for the two far-end QW's can be quantitatively described by a separation energy between this group of two QW's, and another group consists of the rest of the central eight QW's using the mean subband-energy positions of the respective two groups of QW's. This is defined as

$$
E_{D}=\left|E_{9}+\frac{E_{10}-E_{9}}{2}\right|-\left|E_{1}+\frac{E_{8}-E_{1}}{2}\right|
$$




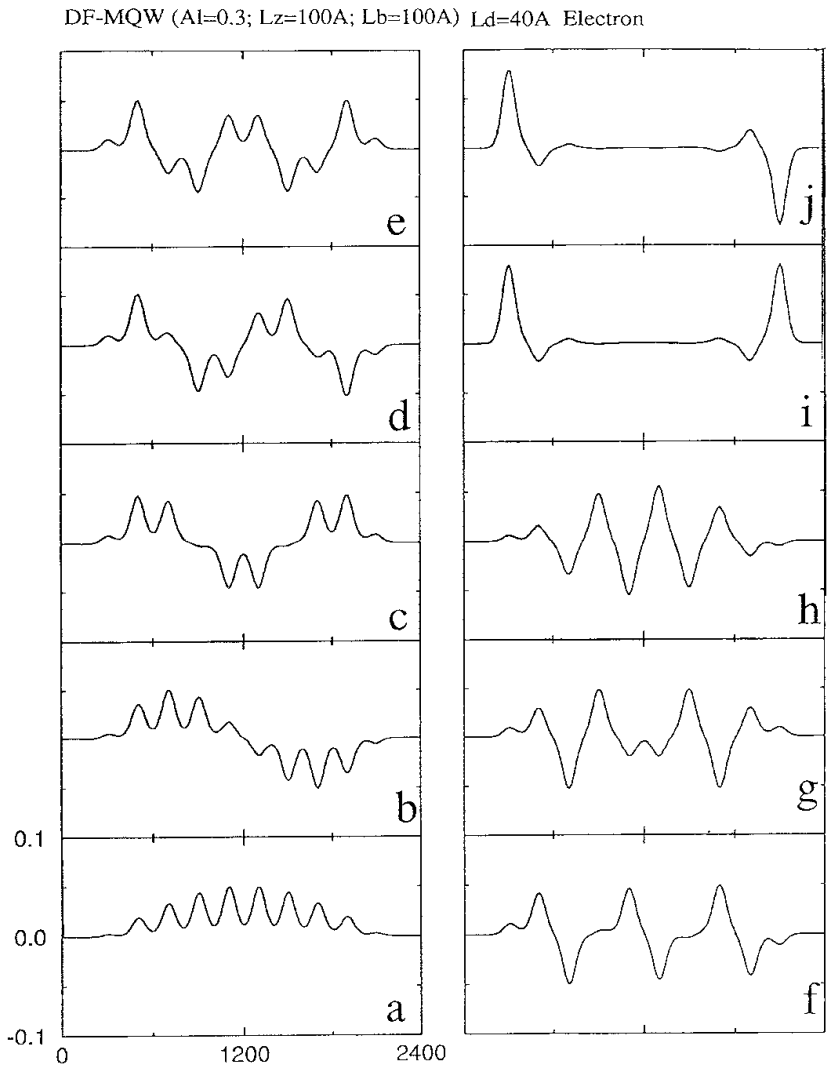

(c)

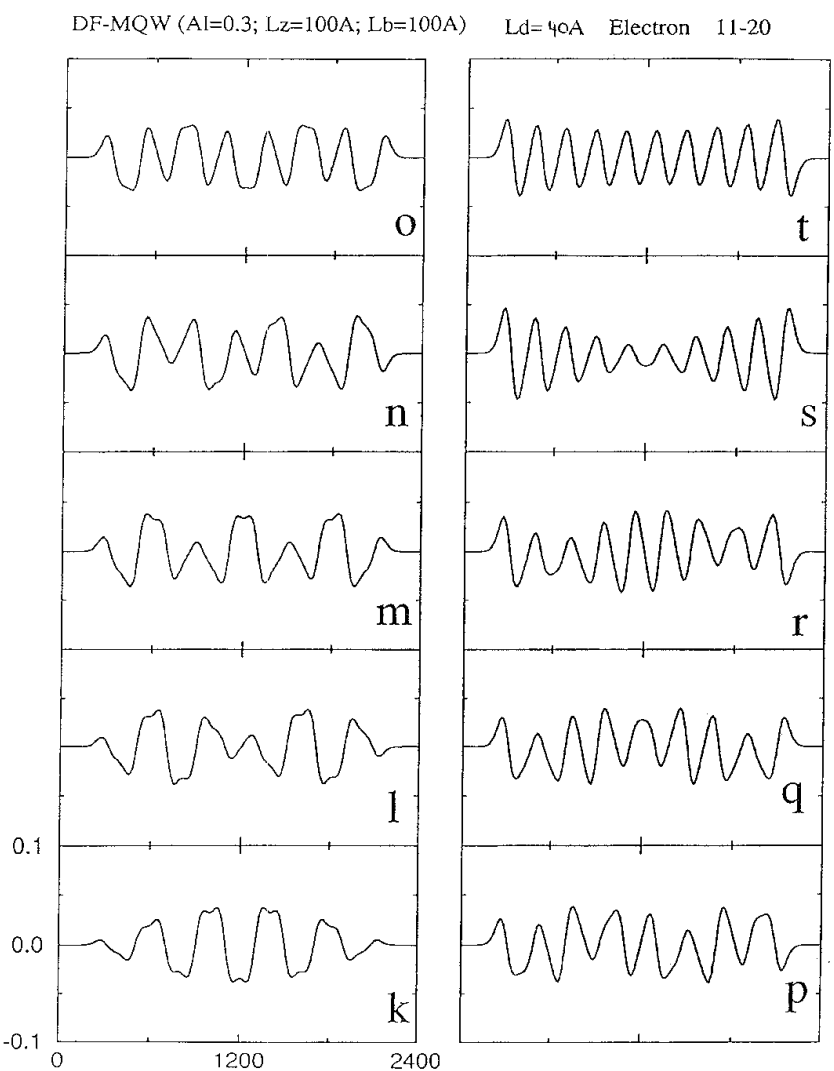

(d)

Fig. 6. (Continued.) The first (lowest energy) 20 eigenstates of electron for three cases where a-j denote the first 10 and $\mathrm{k}-\mathrm{t}$ denote the next 10 . $\mathrm{Al}$ $=0.3, \mathrm{Lz}=100 \AA$, and $\mathrm{Lb}=100 \AA$ for $(\mathrm{c})-(\mathrm{d}) \mathrm{Ld}=40 \AA$.

TABLE III

DF-MQW Subband SPlitTing BAND-Width (meV) FOR the First 10 EIgenstates

\begin{tabular}{||c|c|c|c|c|c|c|c|c|c|}
\hline \multicolumn{2}{|c|}{$\begin{array}{c}\text { first mini- } \\
\text { subband } \\
(10 \text { states })\end{array}$} & \multicolumn{9}{|c|}{$\mathrm{L}_{\mathrm{d}}(\AA)$} \\
\cline { 2 - 10 } & & 5 & 10 & 20 & 30 & 40 & 50 & 60 \\
\hline $\mathrm{C}$ & TBM & 0.019290 & 0.019372 & 0.023485 & 0.077133 & 0.436239 & 2.082465 & 5.540586 & 6.396368 \\
\hline & this work & 0.018509 & 0.018751 & 0.022699 & 0.074347 & 0.408824 & 1.951845 & 5.364641 & 8.126483 \\
\hline HH & TBM & 0.000056 & 0.000050 & 0.000056 & 0.000302 & 0.004903 & 0.069714 & 0.492352 & 1.246786 \\
\hline & this work & 0.000053 & 0.000050 & 0.000056 & 0.000660 & 0.004635 & 0.065599 & 0.462686 & 1.276364 \\
\hline LH & TBM & 0.167349 & 0.170988 & 0.201441 & 0.461179 & 1.409660 & 3.548374 & 5.260472 & 4.027552 \\
\hline & this work & 0.160571 & 0.164462 & 0.193652 & 0.373092 & 1.201327 & 3.249610 & 5.482772 & 6.479593 \\
\hline
\end{tabular}

TBM denotes the tight-binding-method of Bastard and this work represents the full numerical calculation employed here.

for $\ell=1$, and similarly for the other $\ell, \xi$ 's. Although the separation energy $E_{D}$ is only based on the averaged bandedge energy position of the two QW groups, this can serve as a good indicator and measurement of well-shape change for the extreme QW's from the central group of QW's. The delocalized energy separation for the $C(\ell=1,2), H H(\ell=$ $1,2)$, and $L H(\ell=1)$ are shown in Fig. 5(a) and (b). It can be seen that $E_{D}$ clearly increases from approximately zero to an almost constant level for all cases. The separation is always smaller for the lower mini-band as the subbands are tightly confined together. A point worth noting is that for the first $(\ell=1) H H$ minisubband, $E_{D}$ increases as $L_{d}$ increases up to $60 \AA$ and then slightly decreased as $L_{d}$ reaches $100 \AA$. This reflects the low-lying first-subband $(\ell=1)$ levels $H H 9$ and $H H 10$ in the far-end wells where they are still supported by a very shallow well at $L_{d}=60 \AA$ and, as $L_{d}>60 \AA$, they are pushed down by the upper levels $H H(\xi>10)$.

The subband-edge eigenstate wavefunctions also serve as a good characterization of the MQW structure after interdiffusion since it is a "sensitive" function of the change of the well shape. Fig. 6(a) and (b) show the 10 coupled wavefunctions for the first and second subbands, $C(\ell=1,2 ; 1 \leq \xi \leq 10)$, for $L_{d}(\AA)=10,40$, and 60 . The square QW cases are not shown here since they behave in exactly the same manner as a single isolated well due to the 100 - $\AA$-thick barriers. The couplingmode phenomena can be observed here for most of the 


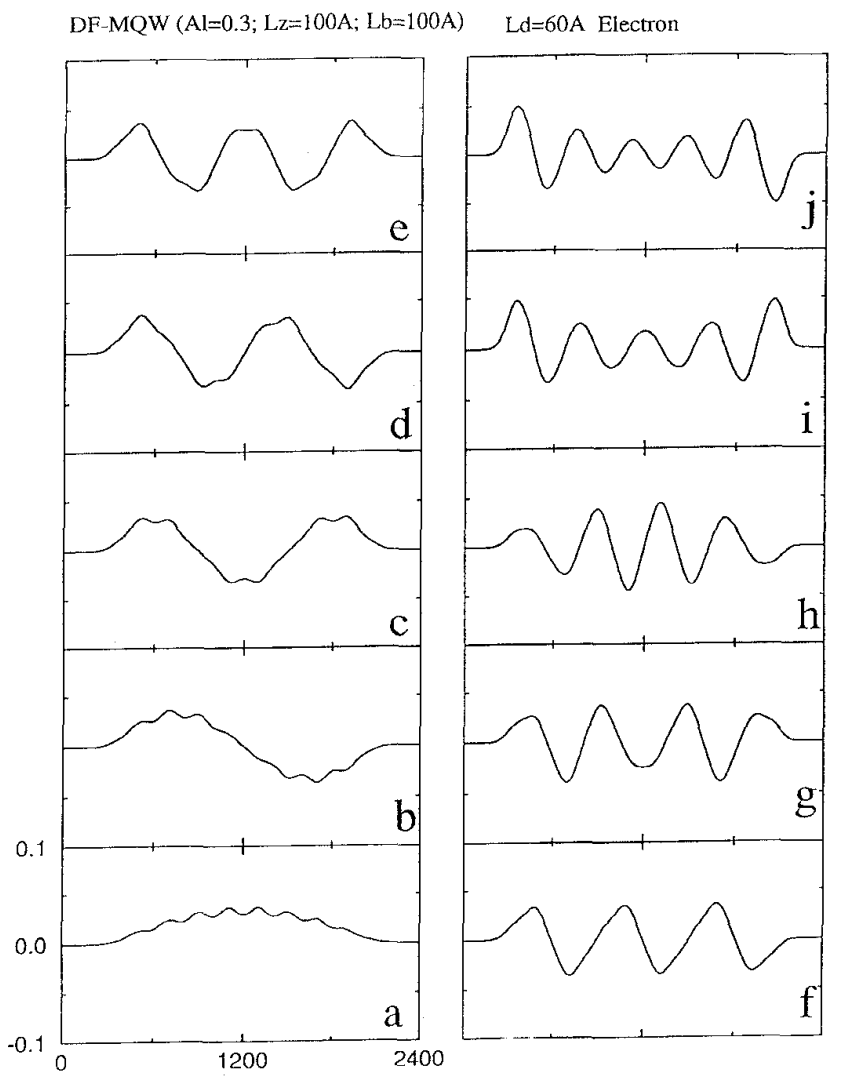

(e)

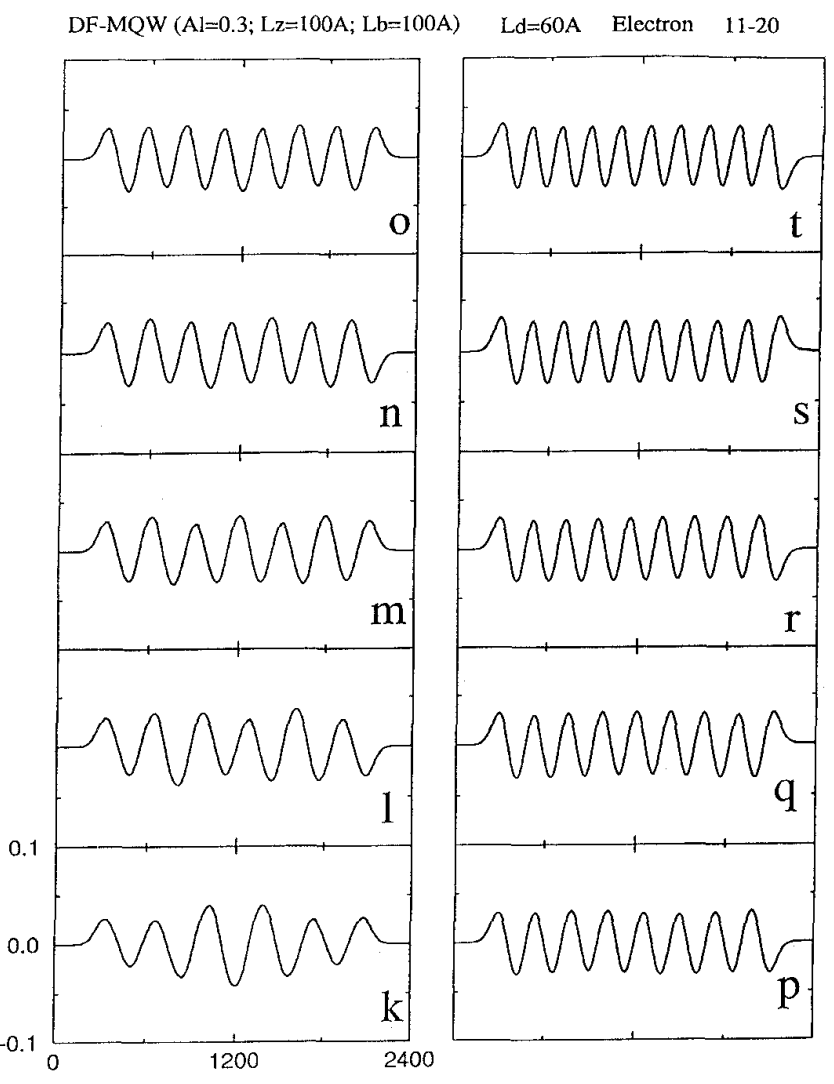

(f)

Fig. 6. (Continued.) The first (lowest energy) 20 eigenstates of electron for three cases where a-j denote the first 10 and $k-t$ denote the next 10 . Al $=0.3, \mathrm{Lz}=100 \AA$, and $\mathrm{Lb}=100 \AA$ for $(\mathrm{e})-(\mathrm{f}) \mathrm{Ld}=60 \AA$.

cases. For a particular subband, there are a total of $N_{w}(=10)$ modes coupled, which are degenerate. It can be seen that these wavefunctions are either symmetric $(\xi=1,3,5,7,9)$ or antisymmetric $(\xi=2,4,6,8,10)$ for all $L_{d}$ values and both minisubbands. Also, the overall wavefunction is enveloped by either an odd or an even function due to the cladding-layer confinement of the entire MQW core, as the wavefunctions are not evenly distributed over each well. Exceptions could be found in the upper states at large interdiffusion extent, such as $(\ell=2 ; \xi \leq 5)$ and $L_{d}=60 \AA$, where the MQW core is almost a uniform material (see Fig. 2), and it behaves like a thick single-QW layer, hence uniformity is restored. However, a peculiar feature of the wavefucntions $\psi_{C 1,9}$ and $\psi_{C 1,10}$, which can be observed in the case of $L_{d}=40 \AA$, is that they are localized to the extremes of the MQW core layers. This confirms the delocalization effect due to nonuniformity of the MQW's after interdiffusion. The nonuniformity of the wavefunctions can also be observed for the cases of $H H\left(L_{d}=\right.$ $40 \AA ; \ell=1,2)$ and $H H\left(L_{d}=20 \AA ; \ell=1\right)$ but not in any of the LH cases considered here. This demonstrates the sensitivity of the energy level to the confinement shape of the well.

\section{Optical Transitions}

The lowest interband transition energy, i.e., bandgap, of the DF-MQW is shown in Fig. 7 as a function of $L_{d}$, and the exact values are given in Table IV for reference. It can be
TABLE IV

DF-MQW BANDGAP ENERGY, $E_{11}=E_{g}\left(L_{d}\right)+E_{C 1,1}+E_{H H 1,1}$

\begin{tabular}{||c|c|}
\hline $\mathrm{L}_{\mathrm{d}}(\AA)$ & $\mathrm{E}_{11}(\mathrm{eV})$ \\
\hline $\mathrm{GaAs}$ & 1.42400 \\
\hline 0 & 1.46340 \\
\hline 5 & 1.46866 \\
\hline 10 & 1.48191 \\
\hline 20 & 1.53381 \\
\hline 30 & 1.59335 \\
\hline 40 & 1.63288 \\
\hline 50 & 1.64974 \\
\hline 60 & 1.65383 \\
\hline 70 & 1.65438 \\
\hline 80 & 1.65444 \\
\hline 90 & 1.65446 \\
\hline 100 & 1.65448 \\
\hline 500 & 1.68822 \\
\hline $\mathrm{Al}_{0.3} \mathrm{Ga}_{0.7} \mathrm{As}$ & 1.84634 \\
\hline
\end{tabular}

seen that during the initial interdiffusion stage the bandgap rises rapidly up to $L_{d}=50 \AA$, which produces a blue shift of 


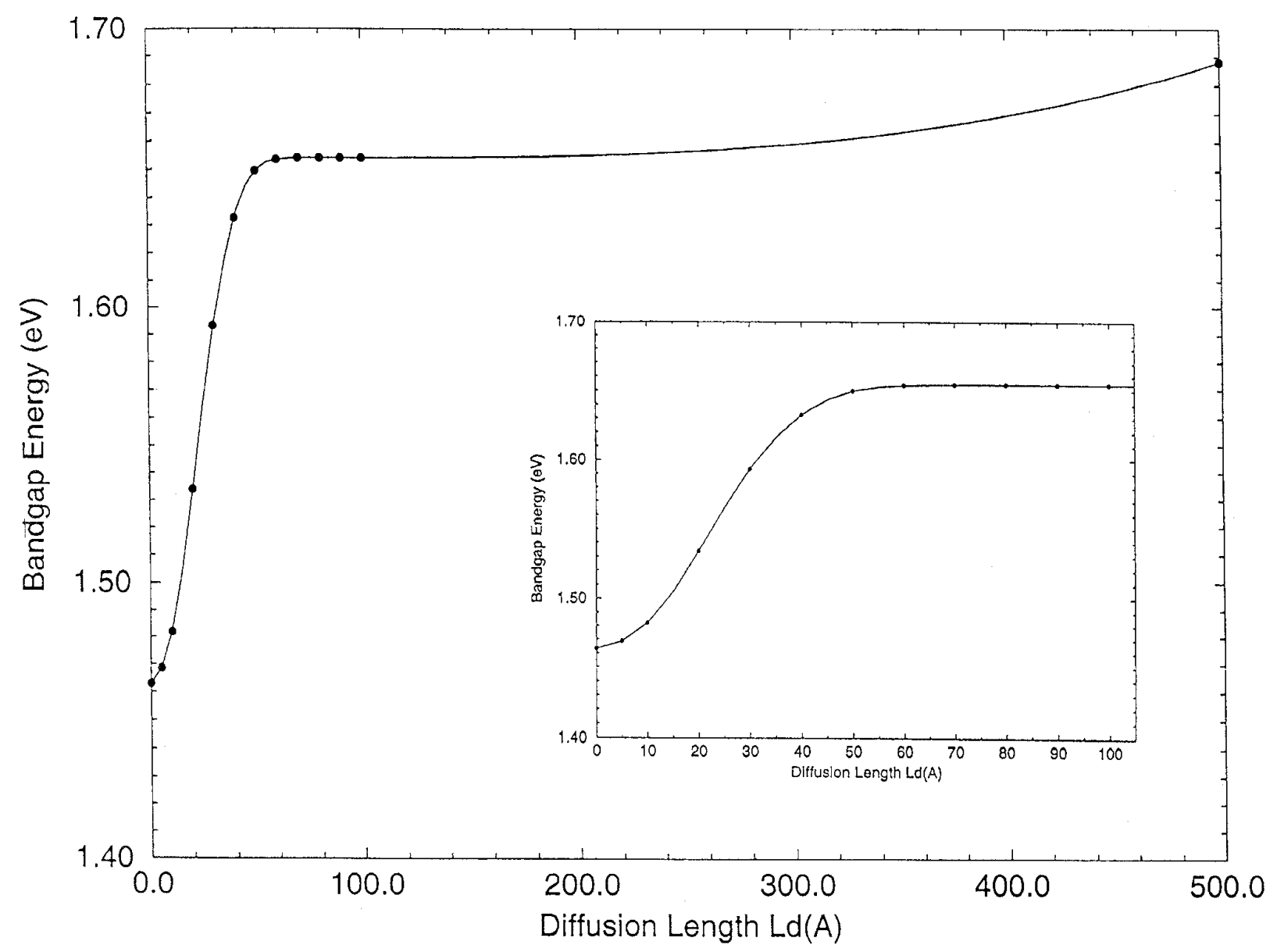

Fig. 7. The DF-MQW band-gap energy as a function of $L_{d}$. Inset shows details of the initial interdiffusion for $L_{d} \leq 100 \AA$.

TABLE $V$

Contrast Ratio (CR) OF A WAVEGUIDE-TYPE LONGITUdinal DF-MQW MOdulator. THE LeNGTH OF THE Modulator is $100 \mu \mathrm{m}$ and TE Polarization is AsSumed

\begin{tabular}{|c|c|c|c|c|}
\hline $\mathrm{Ld}(\AA)$ & Field $(\mathrm{OFF})$ & Field $(\mathrm{ON})$ & $\lambda(\mu \mathrm{m})$ & $\mathrm{CR}(\mathrm{dB})$ \\
\hline 0 & $100 \mathrm{kV} / \mathrm{cm}$ & 0 & 1.4375 & 90.00 \\
\hline 10 & $100 \mathrm{kV} / \mathrm{cm}$ & 0 & 1.4676 & 176.23 \\
\hline 20 & $100 \mathrm{kV} / \mathrm{cm}$ & 0 & 1.5369 & 164.97 \\
\hline
\end{tabular}

$\approx 186 \mathrm{meV}$, after which it then leveled off with only a very gradual increase. This rapid increase is a consequence of the QW effect, and the slow increase is due to a bulk-like material interdiffusion between the cladding layer and the uniform core. The interdiffusion-induced blue shift of the bandgap, $\approx 70 \mathrm{meV}$, at $L_{d}=20 \AA$ compares well with experimental measurement [19] of $61.3 \mathrm{meV}$ for a similar structure at $L_{d} \approx$ $17 \AA .{ }^{4}$ The TE and TM polarized absorption coefficients are shown in Fig. 8(a) and (b), respectively, for several cases of $L_{d}$. The typical signature of the two-dimensional density of states (stepped staircase) can be observed for $L_{d}$ up to $60 \AA$. The magnitude of the first step rises to an absorption of $\approx$

${ }^{4}$ Note that in the experimental data in [21, Fig. 1], a $80-\AA$-wide well and barrier MOW structure is considered, and the parameters used in the determination of the interdiffusion coefficient are not the same as have been used in the present model. Their $950^{\circ} 15 \mathrm{~s}$ RTA corresponds to $L_{d}=17 \AA$.
$5000 \mathrm{~cm}^{-1}$, which is in good agreement with experimental data [33] for the $L_{d}=0$ case. The blue shift of the absorption edge due to interdiffusion is more enhanced in the region of $10 \AA \leq L_{d} \leq 30 \AA$; this corresponds to a band-width of $\approx 0.1 \mathrm{eV}(55 \mathrm{~nm})$ which is half of the $\approx 0.2-\mathrm{eV}(102$ $\mathrm{nm}$ ) width over the range of $0 \AA \leq L_{d} \leq 100 \AA$; which is because of the sharp increase in the MQW core bandgap (the first subband $\ell=1$ ) for this interdiffusion. For small $L_{d}(\leq 10 \AA)$ and $L_{d}(\geq 30 \AA)$, the bandgap energies remain approximately constant, as seen in Fig. 3. These effects are due to the nature of the interdiffusion for small $L_{d}$, where only the well shape has been changed while the depth of the well remains unchanged. However, during the intermediate stages (medium size $L_{d}$ ) not only is the well shape changed but also the depth of the well is reduced, which implies a much more enhanced interband transition energy which gives rise to a much larger blue shift of the bandgap. As the interdiffusion proceeds further, i.e., the latter stages of interdiffusion (large $L_{d}$ ), the well-confinement profile turns into that of a uniform material so that for the interdiffusion process to modify such a thick $(2000-\AA)$ well layer is considerably slower than for the thin $(100-\AA)$ well layers. It is still a single-well structure, but since the well is so thick, the quantum-confinement effects are very small (if they are present at all), and therefore its optical 


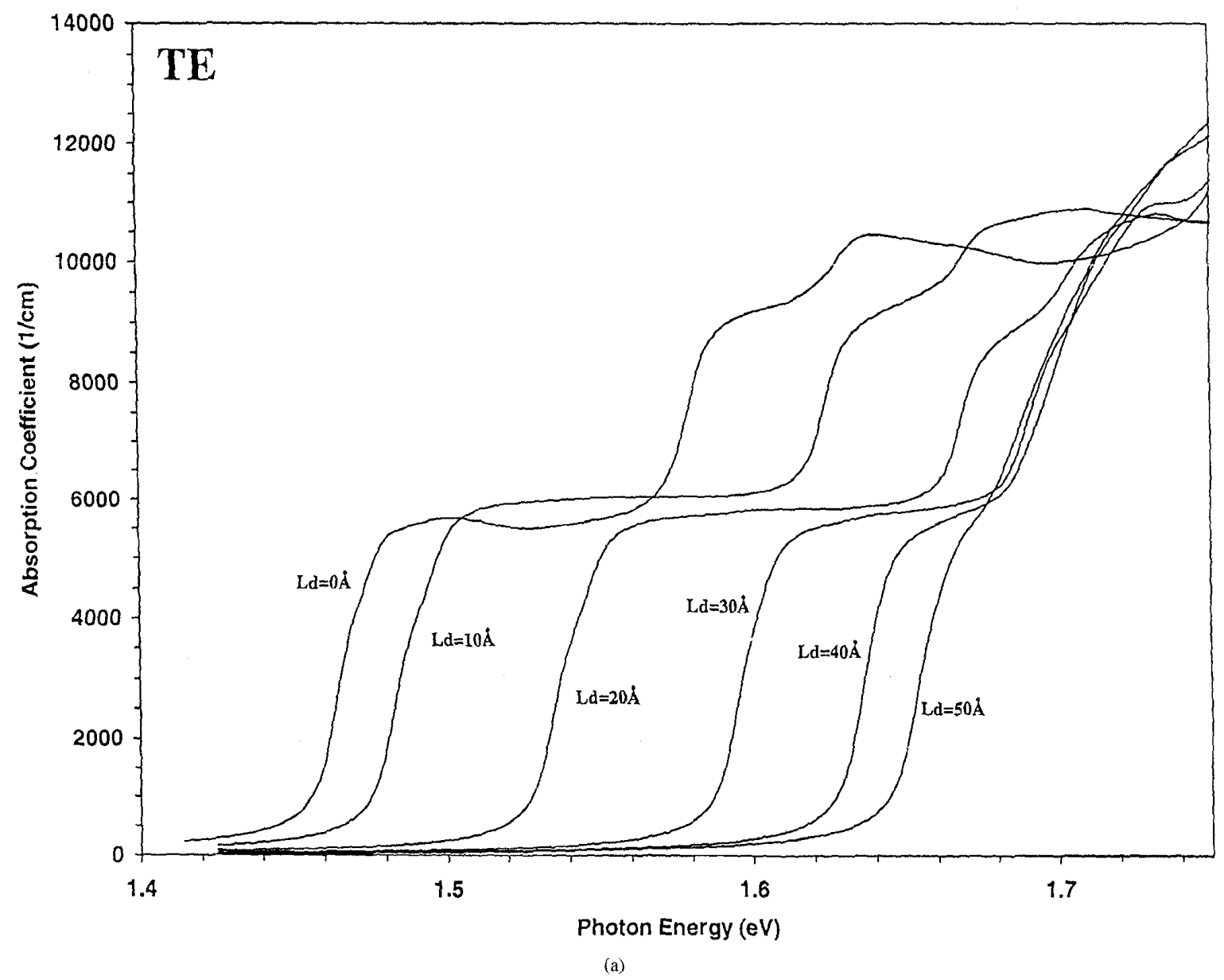

Fig. 8. (a) TE polarized absorption coefficients spectra for $0 \AA \leq L_{d} \leq 80 \AA$.

properties are very insensitive to the well-shape change. These are demonstrated in Fig. 8, where rich features can be observed in the absorption spectrum due to band-mixing, for $\left(L_{d} \leq 60\right.$ $\AA)$, as the interdiffusion proceeds further $\left(L_{d} \geq 60 \AA\right)$, a bulk-like smooth absorption spectrum is produced which is consistent with the theory [10].

\section{Device Implication}

The effect of interdiffusion on the performance of a typical waveguide-type modulator is present here by a quantitative estimate of the conventional contrast ratio (power ON/OFF). For the as-grown MQW $\left(L_{d}=10\right)$, the $\mathrm{CR}$ is $90 \mathrm{~dB}$ at $\lambda=1.438 \mathrm{eV}$, based on the calculation for a modulator with a longitudinal length of $100 \mu \mathrm{m}$. When $L_{d}$ increases to 10 and $20 \AA$, the $\mathrm{CR}$ increase to $176 \mathrm{~dB}(\lambda=1.48$ $\mathrm{eV})$ and $164 \mathrm{~dB}(\lambda=1.54 \mathrm{eV})$, respectively. This implies the electro-absorptive modulation performance improves with interdiffusion. Moreover, the operation wavelength can be varied from $1.438 \mathrm{eV}\left(L_{d}=0\right)$ to $1.54 \mathrm{eV}\left(L_{d}=20 \AA\right)$ which provides a tuning range of almost $60 \mathrm{~nm}$. The only drawback is the increase of propagation loss when $L_{d}$ increased to 10 and $20 \AA$. This loss can be reduced if a larger field is applied where the operation wavelength is shifted further away from the exciton edge and the tuning range can be enlarged if diffusion length is increased. However, if $L_{d}$ is extended to beyond $30 \AA$, the quantum confinement will be diminished as can be seen in Fig. 8(a) and (b) where the two-dimensional nature of the staircase phenomenon is gone at $L_{d}=40 \AA$. Also for electro-absorption, because of losing the two-dimensional absorption, the field strength is restricted to within a small value $(F \leq 70 \mathrm{kV} / \mathrm{cm})$ for cases of large interdiffusion extents $\left(L_{d} \geq 30 \AA\right)$. Thus, in terms of design versatility, this may limit the realization of a wide-bandwidth low-loss modulator. Therefore, optimization of the DFQW structures, including the choice of III-V materials, should be considered, which forms the future work.

\section{CONCLUSION}

A comprehensive analysis of the interwell coupled eigenstates and their interband transitions in the interdiffusion modified $\mathrm{Al}_{0.3} \mathrm{Ga}_{0.7} \mathrm{As}-\mathrm{GaAs} \mathrm{MQW}$ structures has been presented. A full numerical calculation is considered without any 


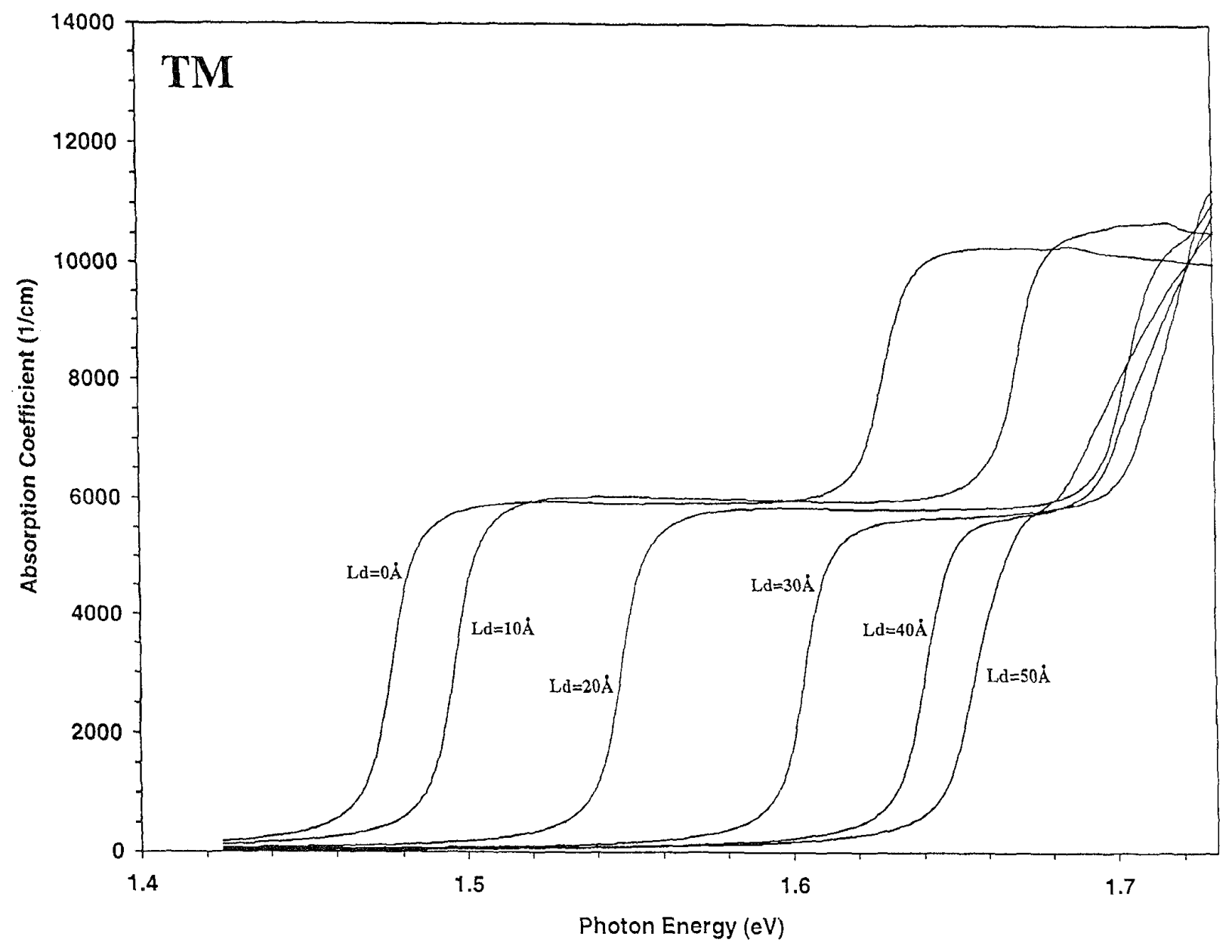

(b)

Fig. 8. (Continued.) (b) TM polarized absorption coefficients spectra for $0 \AA \leq L_{d} \leq 80 \AA$.

approximation or presumption of the eigenstates. The results show that in the initial interdiffusion $\left(L_{d} \leq 10 \AA\right)$ of the quantum-well structure with equal well and barrier thickness of $100 \AA$, the wavefunctions behave as in the case of a single quantum well with almost no well-to-well coupling of the states. However, as interdiffusion proceeds further, the eigenstates in each subband form mini-bands, as is the case of a superiattice, and eventually become a bulk-like material for $\left(L_{d} \geq 100 \AA\right)$. Distortion of the coupled wavefunction can also be observed as a consequence of the nonuniformity of the confined wells at the extremes of the MQW core due to interdiffusion from the thick cladding layers. A rapid increase of the bandgap due to interdiffusion is observed in the initial stage of interdiffusion, which corresponds to an energy blue shift of $\approx 186 \mathrm{meV}$, while the overall shift up to $L_{d}=500 \AA$ is $225 \mathrm{meV}$. The TE and TM polarized absorption coefficients are calculated, including the effect of valence band-mixing. Results show that the blue shifting $(\approx 100 \mathrm{meV}$ ) of the absorption edge is more enhanced in the range of $10 \AA \leq L_{d} \leq$ $30 \AA$, while the absorption strength remains the same. The absorption at $\mathrm{C} 1-\mathrm{HH} 1$ transition for the square quantumwell case is in good agreement with existing experimental data. The interband transition energies and optical properties of interdiffused MQW's have important implications in the design of optoelectronic devices, such as waveguides and modulators. The present results suggest that a large energy shift and, hence, the large refractive-index difference, can be achieved using the as-grown material and that the earlier stage of interdiffusion should produce large refractive-index changes which are suitable for the realization of laterally confined stripe optical waveguides. The spectral-absorption curves also reflect strong two-dimensional quantum-well properties in the beginning of interdiffusion. As a consequence, quantitative estimates for the waveguide-type of modulator suggest a highabsorption loss and low-contrast ratio when interdiffusion is gone too far. Further work is under way in the modeling and analysis of electrooptic effect in these interdiffused MQW structures, together with extensive experiments to support the theoretical analysis.

\section{REFERENCES}

[1] B. L. Weiss, Ed., "Quantum well mixing for optoelectronics," Opt. Quantum Electron., vol. 23, p. S779, 1991. 
[2] O. Wada, A. Furuya, and M. Makiuchi, "Planar, compitible OEIC's based on multiquantum well structures," IEEE Photon. Technol. Lett., vol. 1, pp. 16-18, 1989.

[3] D. G. Deppe and N. Holonyak, Jr., "Atom diffusion and impruity induced layer disordering in quantum well III-V semiconductor herterostructures," J. Appl. Phys., vol. 64, pp. R93-R113, 1988.

[4] D. G. Deppe, L. J. Guido, N. Holonyak, Jr., K. C. Hsieh, R. D. Burnham, R. L. Thornton, and T. L. Paoli, "Stripe-geometry quantum well heterostructure AlxGa1-xAs-GaAs lasers defined by defect diffusion," Appl. Phys. Lett., vol. 49, pp. 510-512, 1986.

[5] J. Werner, T. P. Lee, E. Kapon, E. Colas, N. G. Stoffel, S. A. Schwarz, L. C. Schwartz, and N. C. Andreadakis, "Single and double quantum well lasers with a monolithically integrated passive section," Appl. Phys. Lett., vol. 57, pp. 810-812, 1990.

[6] T. Wolf, C. L. Shieh, R. Engelmann, K. Alavi, and J. Mantz, "Lateral refractive index stip in GaAs/AlGaAs multiple quantum well waveguides fabricated by impurity-induced disordering," Appl. Phys. Lett., vol. 55, pp. $1412-1414,1989$.

[7] J. D. Ralston, W. J. Schaff, D. P. Bour, and L. F. Eastman, "Room-temperature exciton electroabsorption in partially intermixed GaAs/AlGaAs quantum well waveguides," Appl. Phys. Lett., vol. 54, pp. 534-536, 1989.

[8] T. E. Schlesinger and T. Kuech, "Determination of the interdiffusion of $\mathrm{Al}$ and $\mathrm{Ga}$ in undoped (Al,Ga)As/GaAs quantum wells," Appl. Phys. Lett. vol. 49, pp. 519-521, 1986.

[9] K. Kash, B. Tell, P. Grabbe, E. A. Dobisz, H. G. Graighead, and M. C. Tamargo, "Aluminum ion-implantation enhanced intermixing of GaAsAlGaAs quantum well structures," J. Appl. Phys., vol. 63, pp. 190-194, 1988.

[10] E. H. Li, B. L. Weiss, and K. S. Chan, "Effect of interdiffusion of the subbands in an AlGaAs/GaAs single quantum well structure," Phys. Rev. B., vol. 46, pp. 15180-15191, 1992.

[11] E. H. Li and B. L. Weiss, "Exciton optical absorption in a diffusion induced non-square AlGaAs/GaAs quantum well," Proc. SPIE, vol. 1675, pp. 98-108, 1992.

[12] A. T. Meney, "Exciton binding energies and absorption in intermixed GaAs-AlGaAs quantum wells," J. Appl. Phys., vol. 72, pp. 5729-5734, 1992.

[13] E. H. Li, K. S. Chan, B. L. Weiss, and J. Micallef, "The quantumconfined stark effect in an interdiffusion induced quantum well," Appl. Phys. Lett., vol. 63, no. 4, pp. 533-535, 1993.

[14] E. H. Li and B. L. Weiss, "The birefringence properties of interdiffusion induced AlGaAs/GaAs quantum well," Electron. Lett., vol. 28, no. 23, pp. 2114-2115, 1992.

[15] E. H. Li, B. L. Weiss, K. S. Chan, J. Micallef, "The polarization dependent refractive index of an interdiffusion induced $\mathrm{AlGaAs} / \mathrm{GaA}$ quantum well," Appl. Phys. Lett., vol. 62, no. 6, pp. 550-552, 1993.

[16] E. H. Li and B. L. Weiss, "The optical properties of AlGaAs/GaAs hyperbolic quantum well structures," J. Appl. Phys, vol. 70, no. 2, pp. 1054-1056, 1991.

[17] _ "Analytic calculation of subbands and absorption coefficients of AlGaAs/GaAs hyperbolic quantum well," IEEE J. Quantum Electron. vol. 29 , no. 2 , pp. 311-321, 1993

[18] _ "The dielectric properties of non-square AlGaAs/GaAs single quantum well at photon energies below the bandgap," Appl. Phys. Lett. vol. 59, no. 25, pp. 3312-3314, 1991.

[19] J. D. Ralston, S. O'Brien, G. W. Wicks, and L. F. Eastman, "Roomtemperature exciton transitions in partially intermixed GaAs/AlGaAs superlattices," Appl. Phys. Lett., vol. 52, pp. 1511-1513, 1988.

[20] J. D. Ralston, W. J. Schaff, D. P. Bour, and L. F. Eastman, "Roomtemperature exciton electroabsoption in partially intermixed GaAsAlGaAs quantum well waveguides," Appl. Phys. Lett., vol. 54, pp. 534-536, 1989

[21] J. D. Ralston, M. Ramsteiner, B. Dischler, M. Maier, G. Brandt, P. Koidl, and D. J. As, "Intersubband transitions in partially interdiffused GaAs/AIGaAs multiple quantum-well structures," J. Appl. Phys., vol. 70 , pp. $2195-2199,1991$.

[22] M. Ghisoni, P. J.S tevens, G. Parry, and J. S. Roberts, "Post-growth tailoring of the optical properties of GaAs/AlGaAs quantum well structures," Opt. Quantum Electron., vol. 23, pp. S915-924, 1991.

[23] J. Lee, T. E. Schlesinger, and T. F. Kuech, "Interdiffusion of Al and Ga in (Al,Ga)As/GaAs superlattices," J. Vac. Sci. Technol. B, vol. 5, pp. I $187-1190,1987$.

[24] A. T. Meney, "Effects of impurity induced disorder on the index of refraction in GaAs-AlGaAs quantum wells," Superlatt. Microstruct., vol. 11, pp. 47-53, 1992.

[25] J. Crank, The Mathematics of Diffusion. 2nd ed. Oxford, U.K.: Oxford Univ., 1975, p. 15
[26] K. P. Homewood and D. J. Dunstan, "Optical characterization of thermal mixing in quantum wells and heterosturctures using a Green's function model," J. Appl. Phys., vol. 69, pp. 7581-7584, 1991.

[27] D. J. BenDaniel and C. B. Duke, "Space-charge effects on electron tunneling," Phys. Rev., vol. 152, pp. 683-692, 1966.

[28] K. S. Chan, "The effects of the hole sub-band mixing on the energies and oscillator strengths of excitons in a quantum well," J. Phys. C: Solid State Phys., vol. 19, L125-130, 1986.

[29] F. Bassani and G. P. Parravicini, Electronic States and Optical Transitions in Solids. Oxford: Pergamon, 1975, p. 154.

[30] J. S. Weiner, D. S. Chemla, D. A. B. Miller, H. A. Haus, A. C. Gossard, W. Weigmann, and C. A. Burrus, "High anisotropic optical properties of single quantum well waveguides," Appl. Phys. Lett., vol. 47, pp. $664-667,1985$.

[31] E. S. Koteles, B. Elman, R. P. Holmstrom, P. Melman, J. Y. Chi, X. Wen, J. Powers, D. Owens, S. Charbonneau, and M. L. W. Thewalt, "Modification of the shapes of GaAs/AlGaAs quantum wells using rapid thermal annealing," Superlatt. Microstruc., vol. 5, pp. 321-325, 1989.

[32] G. Bastard, "Structure de bandes, niveaux d'impureté et effets Stark dans les super-réseaux," Acta Electron., vol. 25, pp. 147-161, 1983.

[33] D. M. Huang, J. I. Chyi, and H. Morkoç, "Carrier effects on the excitonic absorption in GaAs quantum-well structures: Phase-space filing," Phys. Rev. B, vol. 42, no. 8, pp. 5147-5153, 1990.

[34] E. S. Koteles, "A purely spectroscopic technique for determining energy band offsets in quantum wells," Proc. Mat. Res. Soc., vol. 204, pp 90-104, 1992.

[35] D. E. Aspnes, S. M. Kelso, R. A. Logan, and R. Bhat, "Optical properties of AlGaAs," J. Appl. Phys., vol. 60, pp. 745-767, 1986.

[36] S. Adachi, "GaAs, AlAs, and AlGaAs: Material parameters for use in research and device applications," J. Appl. Phys, vol. 58, pp. R1-R29, 1985.

[37] ㄴ. "Hole effective mass in AlGaAs," in EMIS Dataviews (series no. 7), London: INSPEC, 1992).

[38] G. Lengyel, K. W. Jelley, and R. W. H. Engelmann, "a semi-empirical model for electroabsorption in GaAs/AIGaAs multiple quantum well modulator structures," IEEE J. Quantum Electron., vol. 26, no. 2, pp. 296-304, 1990.

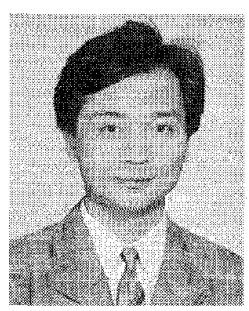

E. Herbert Li (S'87-M'88-SM'95) received the M.Phil. degree in applied mathematics and elec tronic engineering from the University of Hong Kong in 1990 and the $\mathrm{Ph} . \mathrm{D}$. degree in electronic engineering from the University of Surrey, U.K., in 1992.

He worked for the Kirsten Aeronautical Laboratory, Seattle, WA, from 1979-1980. In 1981, he joined K K Engineering, Co., Hong Kong, as an Engineer. In 1986, he joined Micro System, Hong Kong, as a Manager. In 1988, he became a Member of Faculty at the City University of Hong Kong. In 1990, he joined the National Ion Implantation Facility at the Department of Electrical and Electronic Engineering (EEE), University of Surrey, as a Research Project Leader, where he was until 1993. He is currently a Member of Faculty and Leader of the Optoelectronics Group, which consists of a team of 15 researchers, at the Department of EEE, University of Hong Kong. He is a Visiting Full Professor at Shandong University, China, and also holds visiting faculty appointments at the University of Surrey and City University of Hong Kong His current research interests are concerned with optoelectronic-device fabrication, modeling and characterization, interdiffusion-induced modification of quantum-well structures for the integration of optoelectronic devices, acousticoptic devices for slow-rate switching applications, photovoltaic devices, and the development of optical ATM switching systems. Since 1990, he has published over 80 international technical papers and 2 monographs. He is an Editor of the International Journal Of Optoelectronics.

Dr. Li is a member of the Hong Kong IEEE CAS/COM Join Chapter Committee. He served on the Technical Program Committee of the IEEE TENCON '95, and on both of the Technical Program and Internatioal Advisory Committees of OECC '96, Japan. Dr. Li is an Honorary Life Advisor of Pi Mu Epsilon (USA). He received the L. Langham Thompson Premium Prize om 1992 from IEE and the Distinguished Pioneering Projects Award in 1989 from HKCSS. His biography is published in Who's Who in the World and Who's Who in Science and Engineering. 
Bernard L. Weiss (S'72-M'74-SM'92) received the B.Sc. degree in electrical engineering and the Ph.D. degree in solid-state electronics from the University of Newcastle-upon-Tyne, U.K, in 1971 and 1974, respectively.

He was awarded an SRC Personal Postdoctoral Research Fellowship for the study of anodic oxides on GaAs in 1975, and in 1977, he moved to University College, London, U.K., as a Research Fellow, where he worked on acoustic non-destructive testing. In 1979, he was appointed a Lecturer in electronic engineering at Surrey, where he setup research in integrated optical devices in $\mathrm{LiNbO}_{3}$. He was promoted to Senior Lecturer and Reader in 1986 and 1993, respectively, and his research activities are in the modeling of optical properties of III-V semiconductor quantum-well structures and devices, and optoelectronic devices in silicon-based materials. In 1991, he spent six months at the University of Cincinnati, $\mathrm{OH}$, working on the characterization and optical waveguides in quantum-well structures. He was a Visiting Professor at the Technische Hochschule Darmstadt in 1994-1995 and is currently a Visiting Reader at the University of Hong Kong. In 1996, he was promoted to Professor of Microelectronics, where his interests are now in integrated optical devices and circuits in glass and $\mathrm{SiGe}$, the modeling and characterization of III-V semiconductor quantum-well structures and micromechanical devices.

Dr. Weiss is a Fellow of the IEE and the Institute of Physics. He is chairman of the IEE Scholarships Committee, a member of several other IEE committees, and editor of the IEE EMIS book series.

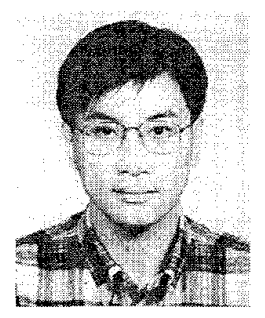

K.-S. Chan (M'88) received the B.Sc. and Ph.D. degrees from the University of Hong Kong in 1981 and 1984, respectively:

From 1984 to 1986, he was a Research Fellow in semiconductor physics at the University of Oxford, Oxford, U.K., working with Sir Roger Elliot. During the same period, he collaborated with the semiconductor research group at Philips Research Laboratory, Redhill; U.K., in studying the optical properties of semiconductor quantum-well structures. From 1986 to 1988 , he was with the Semiconductor Research Group at the Department of Physics, University of Nottingham, Nottingham, U.K. He joined the City University of Hong Kong in 1988, where he is now an Associate Professor and the Course Leader of the B.Sc. degree program in applied physics. He was a Technical Advisor in 1992 to Maxford Technology, Ltd., supervising the development of sputtering technology for decorative coating. His current research focuses on the optical and transport properties of semiconductor heterostructures and their applications in optoelectronics. 